\title{
A numerical study of detonation diffraction
}

\author{
By MARCO ARIENTI ${ }^{1}$ AND J. E. SHEPHERD ${ }^{2}$ \\ ${ }^{1}$ United Technologies Research Center, East Hartford, CT 06108, USA \\ ${ }^{2}$ Graduate Aeronautical Laboratories, California Institute of Technology, Pasadena, \\ CA 91125 , USA
}

(Received 10 March 2003 and in revised form 15 October 2003)

An investigation of detonation diffraction through an abrupt area change has been carried out via a set of two-dimensional numerical simulations parameterized by the activation energy of the reactant. Our analysis is specialized to a reactive mixture with a perfect gas equation of state and a single-step reaction in the Arrhenius form. Lagrangian particles are injected into the flow as a diagnostic tool for identifying the dominant terms in the equation that describes the temperature rate of change of a fluid element, expressed in the shock-based reference system. When simplified, this equation provides insight into the competition between the energy release rate and the expansion rate behind the diffracting front. The mechanism of spontaneous generation of transverse waves along the diffracting front is carefully analysed and related to the sensitivity of the reaction rate to temperature. We study in detail three highly resolved cases of detonation diffraction that illustrate different types of behaviour, super-, sub- and near-critical diffraction.

\section{Introduction}

Detonations are supersonic combustion waves with a strong lead shock front. The shock wave ignites the reactive material, and the exothermic stage of the reactions creates volume expansion that pushes the shock into fresh reactants. Detonations diffracting from a planar to a cylindrical (or spherical) geometry through an abrupt area change experience expansion waves that propagate into the partially burnt reactants behind the wavefront.

One of the key features of this process is the propagation of the signal generated by the expansion waves emanating at the corner. As shown in figure 1(a), the disturbance is propagating at the local acoustic speed $c$ while being convected downstream at a speed $u$. The undisturbed front moves at a constant speed $D$. The angle between the disturbance trajectory and the normal of the undiffracted shock, $\alpha$, can be found by carrying out a Huygen's construction for the wavefront of a sound wave. As discussed in Skews (1967), the wavefront is a circle, of radius $c \Delta t$, whose origin is at point $O$ translated downstream from the corner a distance $u \Delta t$. From the geometric construction, we have

$$
\tan \alpha=\frac{v}{D}=\frac{\sqrt{c^{2}-(D-u)^{2}}}{D} .
$$

In the non-reactive case, the values $u$ and $c$ are evaluated from the post-shock state behind the undisturbed shock. In the reactive case, a finite transverse signal speed is observed in corner-turning experiments with Chapman-Jouguet (CJ) detonations (Schultz 2000). This indicates that acoustic disturbances must propagate in the 

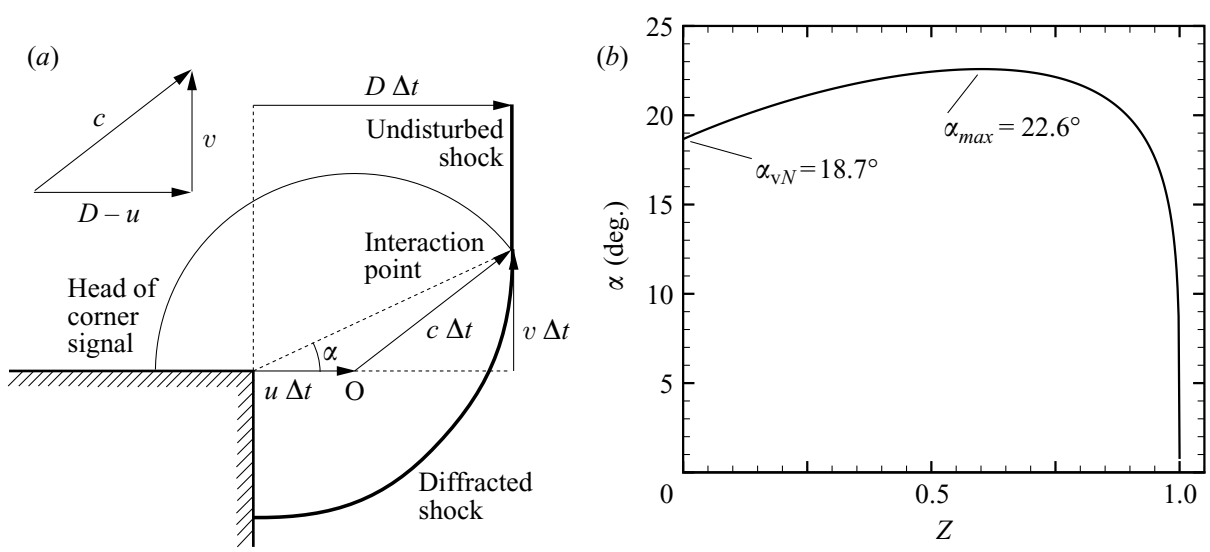

FIGURE 1. (a) Schematic of a diffracting shock (Skews' construction) in non-reactive gas. (b) Disturbance angle plotted vs. progress variable $Z$ for a $Z N D$-CJ detonation. $Z=0$ corresponds to post-shock conditions, $Z=1$ corresponds to the end of the reaction zone.

reaction zone, between the sonic plane (where $D-u=c$ by definition) and the lead shock. Disturbance angles $\alpha$ were measured by Schultz (2000) from a sequence of schlieren images in sub-critical detonation diffraction of hydrocarbon mixtures and hydrogen mixtures. When these results were compared with values computed from the corresponding Zel'dovich-von Neumann-Doering (ZND) profiles (see, for instance, figure $1 b$ ), the angles corresponding to the maximum disturbance velocity were found to be in good agreement with the experimental measurements.

The sensitivity of chemical reactions to post-shock conditions is the second key feature in detonation diffraction. For a single-step reaction model of order $n_{r}$, the rate of reaction of a fluid particle can be described by

$$
\frac{\mathrm{D} Z}{\mathrm{D} t}=k \rho^{n_{r}-1}(1-Z) \exp \left(-\theta T_{v N} / T\right),
$$

where $Z$ monitors the reaction progress (from 0 to 1 ), $\rho$ is the density, $T_{v N}$ is the von Neumann temperature in the ZND profile, and $k$ is a proportionality parameter setting the length scale of energy release. The sensitivity of the chemical kinetics is expressed in the nonlinear term by the reduced activation energy $\theta$, the key parameter in the work presented here.

Since the shock is weakened by the interaction with expansion waves from the corner, the post-shock temperature can be significantly smaller along the diffracting front than in the reference ZND profile, and the reaction process can be quenched or substantially delayed. If the reaction does not take place or else happens far behind the shock front, the reduced amount of energy released into the flow results in a further decay of the detonation speed. As the shock strength diminishes, incoming reactants are less compressed, and this, in turn, increases the ignition delay. Depending on the sensitivity of the reactions to temperature and density changes and on the strength of the rarefaction, the detonation will eventually either be re-established (super-critical diffraction) or cease to propagate (sub-critical diffraction). The conditions that control the transition from super-critical to sub-critical diffraction identify the near-critical state. With all other conditions held constant, the detonation fails for a tube diameter smaller than a critical value (Zel'dovich, Kogarko \& Simonov 1956). 


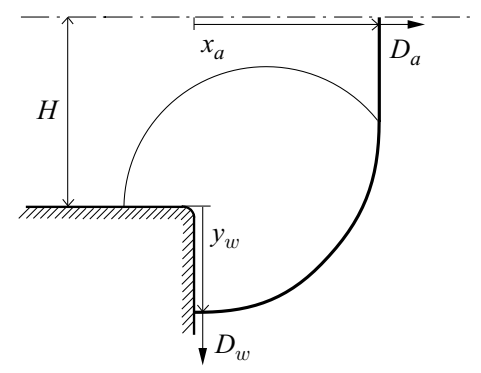

FiguRE 2. Detonation diffraction around a corner. $-\cdot-$, the plane of symmetry of the channel; $H$, the channel half-width. Also shown are the distances measured along the plane of symmetry, $x_{a}$, and along the corner wall, $y_{w}$.

There is an empirical correlation between critical diameter and detonation cell width, $\lambda$ (Mitrofanov \& Soloukhin 1965; Edwards, Thomas \& Nettleton 1979; Knystautas, Lee \& Guirao 1982; Moen et al. 1982; Shepherd et al. 1986; Desbordes 1988). The survey by Guirao, Knystautas \& Lee (1987) for hydrogen-air mixtures indicates that detonation is re-established if the tube diameter is greater than $13 \lambda$. For rectangular orifices with large aspect ratio, the detonation is re-established if the smallest side of the orifice is larger than $3 \lambda$. A recent review of the available detonation diffraction literature can be found in Schultz (2000). Despite the large amount of experimental data, a quantitative theory to predict the critical tube diameter is still lacking.

In two dimensions, an extensive series of simulations with two-step reaction kinetics was performed by Jones et al. $(1990,1991,1995)$ and Oran et al. $(1992,1993)$. Their aim was to reproduce the diffracting patterns in detonation transmission experiments by Liu et al. $(1987,1988)$. The role of detonation cellular structure in detonation diffraction was further investigated by Jones et al. (1996, 2000) and Li \& Kailasanath (2000), through simulations that were found in agreement with the $3 \lambda$ rule. In three dimensions, computations with single-step Arrhenius kinetics were carried out by Williams, Bauwens \& Oran (1996). Their results suggest that vorticity, providing a strong coupling mechanism between perpendicular transverse modes, can be a trigger mechanism for the production of new transverse waves.

In the problem we investigated, detonation diffraction takes place around a sharp corner with an interior angle of $90^{\circ}$ (figure 2). An instance of this situation is encountered when a detonation tube or channel opens into a larger volume. If we assume an unbounded volume, then the only geometric parameter is the exit diameter of the tube, or, in two dimensions, the channel half-width, $H$. Dimensional analysis for the singlestep reaction model in the Arrhenius form leads to the following dependence for the critical channel half-width,

$$
\frac{H_{c}}{\Delta_{1 / 2}}=g\left(\frac{Q}{R_{g} T_{0}}, \gamma_{p}, \gamma_{r}, f, \theta, n_{r}\right)
$$

where $Q$ is the heat of reaction, $\gamma_{r}$ and $\gamma_{p}$ are the reactant and product specific heat ratios, and $f=D / D_{C J}$ is the overdrive of the detonation in the channel. $R_{g}$ is the mixture gas constant and $T_{0}$ is the uniform temperature ahead of the shock. The reaction characteristic length, $\Delta_{1 / 2}$, is defined as the distance, in the reference ZND wave, between the shock $(Z=0)$ and the point where $Z=1 / 2$. Our study is further specialized by setting $f=1, \gamma_{p}=\gamma_{r}$ and $n_{r}=2$. The reference reduced activation 
(a)

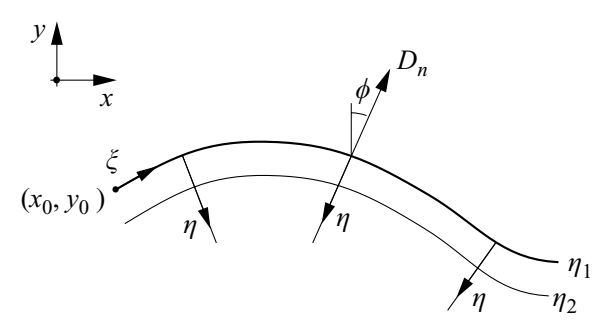

(b)

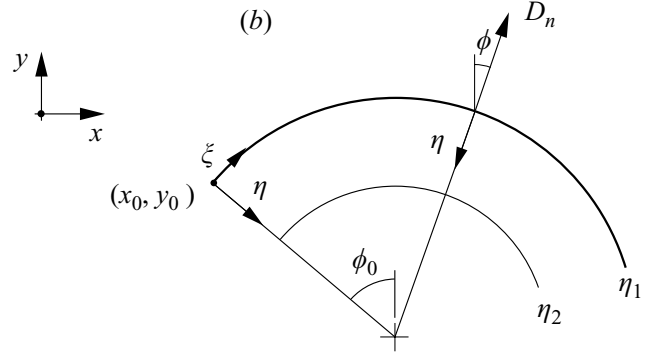

FIGURE 3. (a) Intrinsic coordinates $\xi$ and $\eta$ for an arbitrary front, and $(b)$ specialized to a cylindrical front.

energy $\theta$ is normalized by the von Neumann temperature at CJ conditions and is labelled $\theta_{C J}$.

The concept of shock decoupling from the reaction zone is the simplest idea used to explain the behaviour of a diffracting detonation front. In $\S 2$, we extend to an arbitrary wavefront the equation framework used in the study of direct initiation of spherically symmetric detonations by Eckett, Quirk \& Shepherd (2000). The numerical implementation of the equations of fluid motion, and the algorithms used for flow diagnostics, are described in $\S 3$. In $\S 4$, we examine three cases of detonation diffraction that illustrate different types of behaviour, super-, sub- and near-critical diffraction. The results are discussed in $\S 5$.

\section{Reaction zone structure equations}

\subsection{Governing equations}

Ignoring viscosity, heat transfer, diffusion, radiation and body forces, the governing equations for a compressible reacting flow are the reactive Euler equations completed by a thermal equation of state (see, for instance, Fickett $\&$ Davis 1979). The equation of state for a mixture of perfect gases is written in non-dimensional form as

$$
P=\rho T \text {. }
$$

Variables are non-dimensionalized by taking the uniform conditions upstream of the shock as a reference. From this point onward, dimensional variables are indicated by a tilde. Distance is scaled by $\tilde{\Delta}_{1 / 2}$, and velocity is scaled by the reference particle velocity $\tilde{u}_{0}=\left(\tilde{R}_{g} \tilde{T}_{0}\right)^{1 / 2}$. The mixture gas constant is

$$
\tilde{R}_{g}=\frac{\tilde{\mathscr{R}}}{\tilde{W}}=\tilde{\mathscr{R}} \sum \frac{y_{K}}{\tilde{W}_{K}} .
$$

$\tilde{W}_{K}$ and $y_{K}$ are the molar mass and the mass fraction of species $K, \tilde{\mathscr{R}}$ is the universal gas constant, and $\tilde{W}$ is the mixture molar mass. The non-dimensional activation energy is $E_{a}=\theta T_{v N}$.

In two dimensions, the analysis of the Euler equations can be carried out by using intrinsic shock-based coordinates as independent variables. While more cumbersome than the Cartesian description, this approach allows for the study of the reaction zone structure in terms of local flow features, such as shock curvature. The description of the reactive Euler equations in intrinsic coordinates is essential to this work, and it is, therefore, developed in some length in this section.

In intrinsic coordinates (figure 3 ), the variable $\xi$ measures the arclength of the lead shock from a reference point. This point has coordinates $\left(x_{0}, y_{0}\right)$ in the $x-y$ fixed 
Cartesian reference system. Along the shock, the second coordinate $\eta$ is constant and equal to zero. Lines of constant $\eta$ are the loci of points with the same distance from the shock. The angle $\phi$ between the normal to the front and a reference axis, is a dependent variable, $\phi(\xi, t)$. The two-dimensional curvature of the front, $\kappa$, is, by definition, $\kappa=(\partial \phi / \partial \xi)_{\eta, t}$. $D_{n}$ is the detonation velocity normal to the front. As an example of an intrinsic coordinate system, figure $3(b)$ shows the particular case of a cylindrical front with radius $R(t)$. In this simple situation, the relation between $\xi$ and $\eta$ and the cylindrical coordinates $(r$ and $\phi)$ is given by $\xi=\left(\phi-\phi_{0}\right) R$ and $\eta=R-r$, and the trivial result $\kappa=1 / R$ is found.

Conservation of mass, momentum and total energy can be written in intrinsic coordinates as (Bdzil \& Aslam 2000),

$$
\begin{gathered}
\mathscr{L}(\rho)+\left[\left(D_{n}-u_{\eta}\right) \rho\right]_{, \eta}+\rho \frac{u_{\eta} \kappa+u_{\xi, \xi}}{1-\eta \kappa}=0, \\
\mathscr{L}\left(u_{\eta}\right)+\left(D_{n}-u_{\eta}\right) u_{\eta, \eta}=\frac{P_{, \eta}}{\rho}-\frac{u_{\xi}\left(D_{n, \xi}-u_{\xi} \kappa\right)}{1-\eta \kappa}, \\
\mathscr{L}\left(u_{\xi}\right)+\left(D_{n}-u_{\eta}\right) u_{\xi, \eta}=-\frac{P_{, \xi}+\rho u_{\eta}\left(u_{\xi} \kappa-D_{n, \xi}\right)}{\rho(1-\eta \kappa)}, \\
\mathscr{L}(e)+\left(D_{n}-u_{\eta}\right) e_{, \eta}=\frac{P}{\rho^{2}}\left[\mathscr{L}(\rho)+\left(D_{n}-u_{\eta}\right) \rho_{\eta}\right],
\end{gathered}
$$

where $t$ is time and $P$ and $e$ are pressure and specific internal energy. The variables $u_{\eta}$, $u_{\xi}$ are the particle velocity components in the shock normal and transverse direction. We use the notation, $\xi$ and, $\eta$ to indicate a partial derivative with respect to $\xi$ and $\eta$, in this order. The operator $\mathscr{L}$ is defined as

$$
\mathscr{L}=\left.\frac{\partial}{\partial t}\right|_{\xi, \eta}+\left.\left(B+\frac{u_{\xi}-\eta D_{n, \xi}}{1-\eta \kappa}\right) \frac{\partial}{\partial \xi}\right|_{t, \eta} .
$$

Note that, since the intrinsic reference system is time varying, the shock-based partial time derivative differs from the partial time derivative evaluated in a fixed reference. In (2.4), $B$ is the rate of change in arclength with respect to a fixed axis of reference as measured by an observer that is always moving in the shock normal direction (Bdzil \& Stewart 1989). The Lagrangian derivative, computed along the path of a fluid element, can be expressed as

$$
\mathrm{D} / \mathrm{D} t=\mathscr{L}+\left(D_{n}-u_{\eta}\right) \partial / \partial \eta .
$$

For $N$ species, the rate of change of species $K$ of a fluid element is

$$
\frac{\mathrm{D} y_{K}}{\mathrm{D} t}=\Omega_{K}
$$

with the index $K$ varying between 1 and $N$. Using simple thermodynamic relations, the energy equation $(2.3 d)$ may be replaced by the adiabatic change equation (Fickett \& Davis 1979),

$$
\frac{\mathrm{D} P}{\mathrm{D} t}=c^{2} \frac{\mathrm{D} \rho}{\mathrm{D} t}+\rho c^{2} \sum_{K} \sigma_{K} \Omega_{K},
$$

where $c$ is the frozen sound speed. The sum of the thermicity coefficients $\sigma_{K}$ in (2.7) expresses the total pressure change due to chemical reaction at constant volume, and 
is called the thermicity product $\dot{\sigma}$,

$$
\dot{\sigma}=\sum_{K} \sigma_{K} \Omega_{K} .
$$

In this work, we specialize the reaction model to a one-step irreversible reaction, $\mathrm{A} \rightarrow \mathrm{B}$, where the upstream fluid is undiluted species $\mathrm{A}$. The reactant and product are taken to have the same specific heat ratio $\gamma$. The specific internal energies of species $\mathrm{A}$ and $\mathrm{B}$ are

$$
e_{\mathrm{A}}=C_{v} T, \quad e_{\mathrm{B}}=C_{v} T-Q,
$$

where $C_{v}$ is the gas specific heat at constant volume. The caloric equation of state is

$$
e=\frac{1}{\gamma-1} T-Z Q \text {. }
$$

The progress variable $Z$ is defined as the mass fraction of product $\mathrm{B}, Z=y_{\mathrm{B}}=1-y_{\mathrm{A}}$, the thermicity is

$$
\dot{\sigma}=(\gamma-1) \frac{Q}{c^{2}} \frac{\mathrm{DZ}}{\mathrm{D} t},
$$

and the reaction rate is given by (1.2).

\subsection{The Lagrangian derivative of temperature}

Since realistic reaction rates are strongly temperature dependent, the Lagrangian derivative of temperature, $\mathrm{D} T / \mathrm{D} t$, is of particular interest when considering the possibility of detonation failure. By taking the Lagrangian derivative of (2.1), and using the adiabatic change equation (2.7) together with the mass and momentum equations in (2.3), we find the temperature reaction zone structure equation

$$
\begin{aligned}
\left(1-\frac{w_{\eta}^{2}}{c^{2}}\right) C_{p} \frac{\mathrm{D} T}{\mathrm{D} t}= & \frac{1}{\gamma-1}\left(c^{2}-\gamma w_{\eta}^{2}\right) \dot{\sigma}+w_{\eta}^{2} \frac{\kappa\left(D_{n}-w_{\eta}\right)}{1-\eta \kappa} \\
& +w_{\eta}\left(D_{n}-w_{\eta}\right)_{, t}+\frac{P_{, t}}{\rho}+w_{\eta}^{2} \frac{w_{\xi, \xi}}{1-\eta \kappa}-w_{\eta} \frac{w_{\xi}^{2} \kappa}{1-\eta \kappa} \\
& +\frac{w_{\xi}}{1-\eta \kappa}\left(-w_{\eta} w_{\eta, \xi}+\frac{P_{, \xi}}{\rho}\right)+B\left(w_{\eta}\left(D_{n}-w_{\eta}\right)_{, \xi}+\frac{P_{, \xi}}{\rho}\right) \\
& -\frac{\eta D_{n, \xi}}{1-\eta \kappa}\left(w_{\eta}\left(D_{n}-w_{\eta}\right)_{, \xi}+\frac{P_{, \xi}}{\rho}\right)+w_{\xi} w_{\eta} \frac{2 D_{n, \xi}}{1-\eta \kappa}
\end{aligned}
$$

with $w_{\eta}=D_{n}-u_{\eta}$ and $w_{\xi}=u_{\xi}$. $C_{p}$ is the mixture specific heat at constant pressure, $C_{p}=\gamma /(\gamma-1)$. Equation (2.12) has the dimension of energy density per unit time. The right-hand side has terms depending on the thermicity product, the shock curvature, the partial time derivatives of the flow, the transverse divergence $w_{\xi, \xi}$, and a term in $w_{\xi}^{2} \kappa$ that has the appearance of work associated with centripetal motion. The remaining terms in the last two rows of (2.12) are more difficult to interpret.

The two terms containing a partial time derivative in the intrinsic reference frame can be grouped together, and, for the remainder of this work, they will be referred to as 'unsteady terms' or 'unsteadiness' of the fluid particle. Note that in a decelerating wave, such as occurs in detonation diffraction, the unsteady terms are always negative. Thus, the reaction may quench if the wave is decelerating too rapidly.

If the plane of reference is also a plane of symmetry for the flow field, several terms disappear at $\xi=0$. The transverse derivatives vanish, with the exception of $w_{\xi, \xi}$, and 
we obtain

$$
\begin{aligned}
\left(1-\frac{w_{\eta}^{2}}{c^{2}}\right) C_{p} \frac{\mathrm{D} T}{\mathrm{D} t}=\frac{1}{\gamma-1}\left(c^{2}-\gamma\right. & \left.w_{\eta}^{2}\right) \dot{\sigma}+w_{\eta}^{2} \frac{\kappa_{s}\left(D_{s}-w_{\eta}\right)}{1-\eta \kappa_{s}} \\
& +w_{\eta}\left(D_{s}-w_{\eta}\right)_{, t}+\frac{P_{, t}}{\rho}+w_{\eta}^{2} \frac{w_{\xi, \xi}}{1-\eta \kappa_{s}} .
\end{aligned}
$$

In (2.13), $\kappa_{s}(t)$ and $D_{s}(t)$ are the front curvature and shock speed evaluated on the plane of symmetry. The result is the same as that found for a cylindrically symmetric flow with the addition of a transverse divergence term (Eckett et al. 2000). This term is always positive since $w_{\xi}$ is anti-symmetric and no mass flux is allowed at the plane of symmetry. Note that, when the wavefront is convex-upstream, the curvature term is also positive in (2.13) and so it cannot possibly be a source of reaction quenching without the additional presence of unsteadiness.

The relative size and behaviour of the terms in the temperature reaction zone structure equation will be examined numerically by following the path of massless particles injected into the flow. We will refer to these particles as Lagrangian. The goal is to identify the dominant balance in a Lagrangian particle close to ignition failure and to find any simplifying assumption regarding the behaviour of terms in (2.12) or (2.13).

\section{Numerical implementation}

The reactive Euler equations are integrated via operator splitting as an alternated sequence of convective and reaction source steps (Strang 1968). In the convective step, numerical fluxes are computed with Roe's approximate solution of the Riemann problem (Roe 1986). Formal second-order spatial accuracy is obtained via minmod flux limiting, and the scheme is made entropy-satisfying with Harten's entropy fix (Harten 1983). In the reaction source step, at $\rho$ and $e$ constant, the ordinary differential equation

$$
\frac{\mathrm{d} Z}{\mathrm{~d} t}=k \rho(1-Z) \exp \left(-E_{a} / T\right)
$$

is integrated by a second-order time-accurate predictor-corrector scheme. Verification of results and the detailed description of the one-dimensional solver can be found in Eckett (2001). The scheme is extended to two dimensions via standard dimensionby-dimension integration, and is marched in time with the forward Euler integration scheme.

To accelerate the program execution time, the solver is embedded in the Grid Hierarchy Adaptive Computational Engine library, or GrACE (Parashar et al. 1997; Parashar \& Browne 2000). This parallel library operates on partitions of the computational domain that are assigned to different CPUs of a multi-processor computer. Communication between processors is based on the Message Passing Interface (MPI) protocol (see, for instance, Snir et al. 1996).

A schematic diagram of the computational domain is shown in figure 2. For simplicity, a zero-gradient condition is imposed on the flow variables at the inlet (lefthand boundary), even if the flow, initially at CJ conditions, becomes subsonic when the rarefaction signal from the corner moves upstream. The length of the channel is $0.9 \mathrm{H}$ so that the corner expansion is protected from perturbations coming from the inlet in the early phases of detonation diffraction. Reflective boundary conditions are implemented at the plane of symmetry (top boundary) and the simulation is 


$\begin{array}{lllllllll}\theta_{C J} & 0.0 & 1.0 & 2.0 & 2.5 & 3.0 & 3.5 & 3.75 & 4.15 \\ k & 0.2013 & 0.3931 & 0.7757 & 1.077 & 1.551 & 2.204 & 2.632 & 3.509\end{array}$

TABLE 1. Normalized activation energy and proportionality factor.

terminated before the detonation front exits the domain at the right-hand and bottom boundaries.

To remove the singularity introduced in an Euler (inviscid) flow around a sharp corner, the vertex is described by 128 segments as a polygonal boundary, approximating a rounded corner with radius of curvature $r_{c}=1$. The ghost-fluid coupling scheme is used to model the correct reflective boundary conditions at the wall, solving the issue of Cartesian cells that are cut by the polygonal boundary. An extensive description of this level-set based technique can be found in Arienti et al. (2003) in the context of the solution of dynamic fluid-solid coupling problems with complex interfaces. The details of the actual corner shape are, however, unimportant since it is found that, for a sufficiently small radius (compared to the reference reaction length), the flow field is affected only within a distance of a few multiples of $r_{c}$ from the corner (Arienti 2002).

At time $t=0$, the initial solution is a planar ZND-CJ wave travelling from left to right in the inlet channel. The reference state ahead of the shock is $\tilde{P}_{0}=24 \mathrm{kPa}$ and $\tilde{T}_{0}=298 \mathrm{~K}$. The specific heat ratio, $\gamma=1.22$, and gas constant, $\tilde{R}_{g}=274.4 \mathrm{~J} \mathrm{~kg}^{-1} \mathrm{~K}^{-1}$, are estimated for stoichiometric oxyacetylene. These properties and the CJ detonation speed, $\tilde{D}_{C J}=2346.1 \mathrm{~m} \mathrm{~s}^{-1}$ (corresponding to the Mach number 7.422 and the non-dimensional value $D_{C J}=8.204$ ), were evaluated with the program STANJAN (Reynolds 1986). The non-dimensional energy release is $Q=65.81$. To compare consistently the wave profiles computed for different values of $\theta_{C J}$, the proportionality parameter $k$ in the reaction rate formula (3.1) is adjusted so that $\tilde{\Delta}_{1 / 2}$ is the same in all the simulations (see table 1 for a list of values).

An important part of the numerical results that are presented in this work depends on the correct tracking of the detonation front; see Appendix A for more details. Particular care is required to control the error associated with time and space derivatives of the estimated lead shock position. This error is due to the necessary use of interpolation in the shock-tracking procedure and to high-frequency solution oscillations that occur when the wavefront crosses the interface between computational cells.

A second analysis tool is provided by the massless particles that are injected into the flow to register the thermodynamic state and velocity of the surrounding fluid. At each time step, these particles are first advected by the flow-field solution and then used to interpolate the flow-field variables and gradients at their current location. Each particle data stream is separately stored to be post-processed at the end of the simulation. More details on this procedure can be found in Appendix B.

\section{Activation energy studies}

The reduced activation energy $\theta_{C J}$ is the key parameter determining the dynamics of a combustion system described by a one-step Arrhenius rate model. Large values of $\theta_{C J}$ result in a chemical reaction rate that is very sensitive to changes in the thermodynamic state. Small values of $\theta_{C J}$ result in a chemical reaction rate that is 


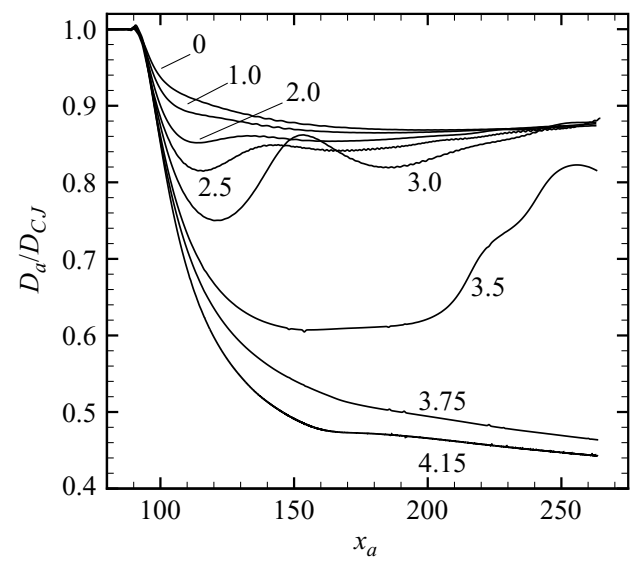

FiguRE 4. Detonation velocity at the plane of symmetry, $D_{a}$, as a function of the distance measured from the vertex, $x_{a}$. The labels are values of the reduced activation energy $\theta_{C J}$, varying from 0 to 4.15 .

almost independent of changes in the thermodynamic state. As a consequence, the diffraction behaviour of detonations modelled with an Arrhenius rate law can vary widely depending on the magnitude of the activation energy. In the present study, a range of values has been examined in order to map out the possible types of diffraction behaviour that can occur with a fixed ratio of reaction zone length to channel height.

Two types of study were carried out. First, a set of coarse-resolution simulations was performed for eight values of $\theta_{C J}$ between 0 and 4.15. Secondly, a set of highresolution simulations was carried out for three selected cases with reduced activation energies of 1, 3.5 and 4.15. All of these simulations were performed with an initially planar ZND wave travelling at the CJ speed before diffracting around the corner. Normal mode stability analysis (Lee \& Stewart 1990) indicates that the neutral stability curve for one-dimensional CJ detonations asymptotes to a constant value $\theta_{C J} \cong 4.74$ for sufficiently large Mach numbers $\left(M_{C J}>6\right)$. Our simulations lie entirely within the range of one-dimensional hydrodynamic stability, allowing the study of purely gasdynamic quenching mechanisms in detonation diffraction.

Computations were carried out over a sufficiently long time to determine the ultimate fate of the detonation wave. The half-width $H$ of the channel in these simulations was fixed at 36.67 reaction half-lengths.

The coarse-resolution studies were performed with 16 grid points per half-reaction zone length, $N_{1 / 2}=16$. This resolution level was convenient since it enabled a complete simulation (on a 4824 by 3752 grid) to be performed in less than 36 wall-clock hours on 48 processors (Pentium III, $1 \mathrm{GHz}$ with $1 \mathrm{~GB}$ of RAM) of the ASAP Linux cluster in the Centre for Advanced Computing Research (CACR) at Caltech.

\subsection{Coarse-resolution studies}

The histories of the shock detonation speeds, $D_{a}$ and $D_{w}$ (shown in figure 2), are plotted as a function of position in figures 4 and 5 . In figure 4 , the shock speed on the plane of symmetry remains constant until the first expansion wave reaches the centre of the channel at about 90 half-reaction lengths from the corner vertex location. The expansion causes the shock speed to decay in all cases, but the long-time behaviour is different depending on the values of reduced activation energy. 


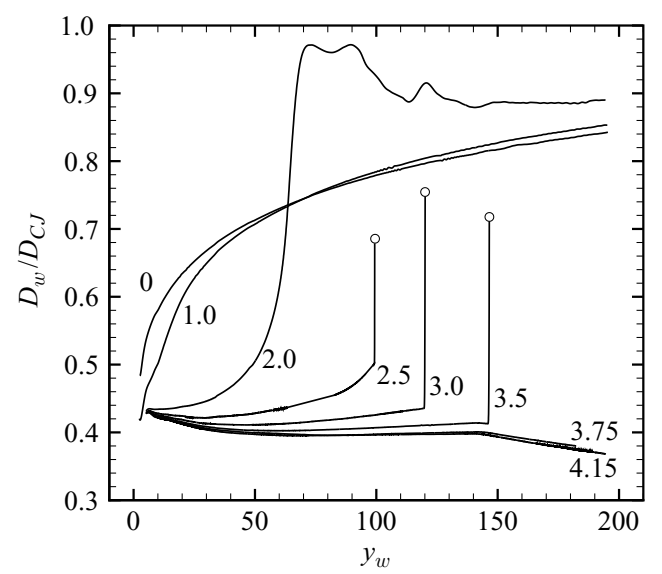

FIGURE 5 . Detonation velocity at the corner wall, $D_{w}$, as a function of the distance from the vertex, $y_{w}$. The labels are values of the reduced activation energy $\theta_{C J}$, varying from 0 to 4.15 .

In figure 5, the shock speed at the wall drops instantly, since the flow around the corner immediately affects the shock front. The very low pressure in the corner region causes the shock to propagate at much lower velocity along the wall than along the plane of symmetry. Overall, in this initial phase of corner diffraction, the behaviour of diffracting detonations is very similar to that observed with non-reacting shock waves.

For longer times, two extreme types of behaviour can be noted for low activation energy and high activation energy. These behaviours resemble the super-critical (low activation energy) and sub-critical (high activation energy) diffraction cases observed in experimental studies of diffraction from tubes. In addition, the cases of intermediate activation energy appear to be similar to the critical case of diffraction from tubes. It is important to keep in mind two key differences between experiments and the present simulations. First, the reduced activation energy is between 4 and 7 for most fueloxidizer combinations (Schultz 2000). Secondly, there are always transverse waves present on the detonation prior to reaching the corner so the correspondence between experiment and present simulations is necessarily inexact. More realistic computations are required in future studies to examine the influence of these two factors.

\subsubsection{Low activation energy}

For $0 \leqslant \theta_{C J} \leqslant 1$, the reaction rate is essentially independent of the thermodynamic state so that the reaction zone length is unaffected by the shock velocity. Since the reaction rate is nearly constant, the detonation will always accelerate after diffraction, reaching the CJ velocity far from the corner. This is similar to the case of super-critical diffraction that is observed in diffraction experiments (Schultz 2000) where the tube is larger than the critical size required for successful detonation transmission.

Upon examination of figures 4 and 5, a simple picture of the low-activation-energy case emerges. The detonation velocity initially decreases owing to the expansion waves created by the flow around the corner, yet, after the initial decay, the wave accelerates and eventually approaches the CJ velocity at a large distance from the corner. The velocity on the plane of symmetry drops slowly to about $88 \%$ of the CJ velocity and then begins to recover after propagating to 200 half-reaction lengths along the plane of symmetry (figure 4). The velocity on the wall drops immediately to $40 \%$ of the CJ value and recovers to about $80 \%$ of $\mathrm{CJ}$ by the time the shock has propagated 200 
half-reaction lengths along the wall (figure 5). The magnitude of the drop in the shock velocity and the rate of acceleration are associated with the competition between the gasdynamic expansion created by corner flow and the energy release immediately behind the shock.

\subsubsection{High activation energy}

For $3.75 \leqslant \theta_{C J} \leqslant 4.15$, the reaction rate is strongly dependent on the thermodynamic state so that the reaction zone length increases rapidly when the shock speed decreases. This causes the reaction zone to decouple from the shock wave, and the reaction rate to essentially drop to zero, after a short distance from the corner vertex. The detonation fails completely and the resulting flow is essentially a non-reactive shock wave. This is similar to the case of sub-critical diffraction that is observed in diffraction experiments where the tube is smaller than the critical size required for successful detonation transmission (Schultz 2000).

Examining figures 4 and 5, we find that there is also a simple pattern of behaviour for this case. The wave velocity on both the plane of symmetry and the wall decreases continuously and reaches very low values, less than $50 \%$ of the CJ value at 200-250 half-reaction lengths from the corner vertex. The dynamics of the wave propagation are essentially those of a non-reactive shock, and the approximate method of Whitham (1974) can be used to find the evolution of the front.

\subsubsection{Intermediate activation energy}

For $2.5 \leqslant \theta_{C J} \leqslant 3.5$, the reaction rate is moderately dependent on the thermodynamic state. The reaction zone length increases as the shock decays, but the accelerating effects of energy release are sufficient to cause the reaction zone length to decrease ultimately in an abrupt fashion. This gives the appearance of a re-ignition event near the wall (figure 5) that propagates back to the centre of the channel. This is similar to the case of critical diffraction that is observed in diffraction experiments where the tube is comparable to the critical size required for successful detonation transmission (Schultz 2000).

The axial and wall velocities show an initial decay to a velocity higher than that observed in the high-activation-energy cases, followed by an acceleration back to velocities similar to the low-activation-energy cases. The acceleration is abrupt at the wall, but more gradual on the plane of symmetry. This is related to the mechanism of transition, discussed in more detail below.

Another way to analyse the flow near the channel centre is to plot wave speed $v s$. curvature (figure 6). A unique $D_{n}-\kappa$ relationship should result if the flow is quasisteady in nature (Bdzil \& Stewart 1989; Yao \& Stewart 1995; Stewart \& Yao 1998). Although the curves of figure 6 have a backward C-shape, their numerical values can be very different from those in the corresponding $D_{n}-\kappa$ curves. These differences are reported in detail in Arienti (2002).

\subsection{High-resolution studies}

Following the results of the coarse-resolution studies, more detailed simulations were performed for selected cases at high resolution. The need for highly resolved computations is crucial in the study of detonation diffraction. When the reaction zone is under-resolved, direct numerical simulations tend to overestimate the wavefront curvature (Menikoff, Lackner \& Bukiet 1996), and poor predictions of detonation wave structure can be expected (Sharpe 2001). The following three simulations were computed with $N_{1 / 2}=22.5$ on a $6570 \times 5858$ grid for $\theta_{C J}=1$ and 3.5 , and on a 


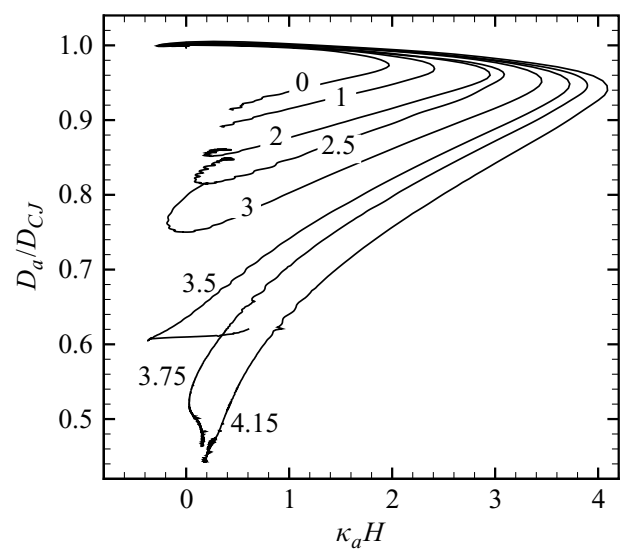

FiguRE 6. Detonation velocity-curvature $\left(D_{n}-\kappa\right)$ diagram at the plane of symmetry of the channel. The labels are values of the reduced activation energy $\theta_{C J}$, varying from 0 to 4.15 .

(a)

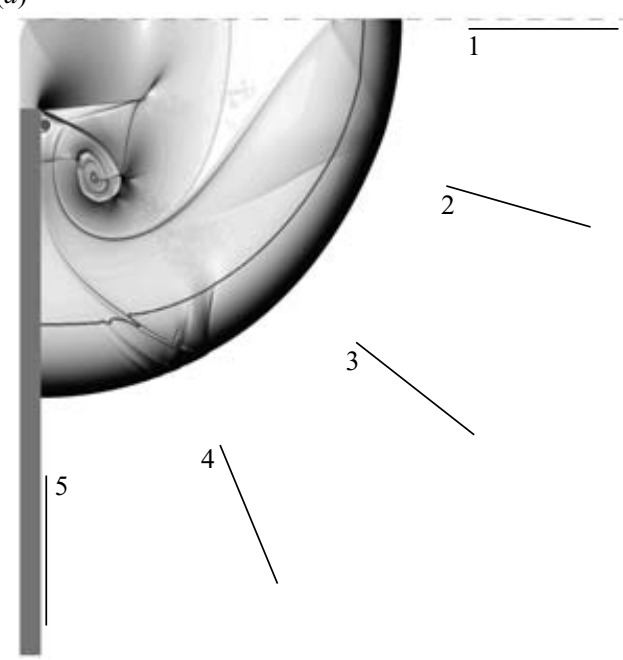

(b)

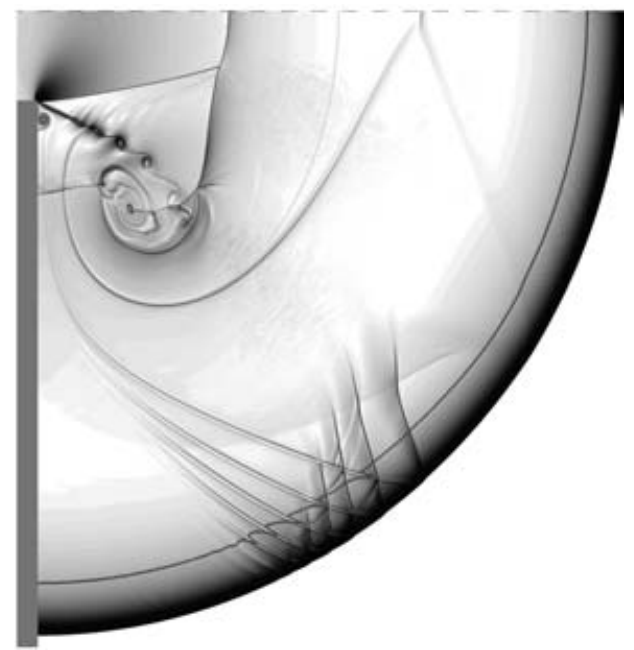

FIgURE 7. Numerical schlieren images for the case $\theta_{C J}=1$. (a) $t=21.15$; (b) 35.79 . The solid line is the locus of $95 \%$ product.

$6750 \times 5100$ grid for $\theta_{C J}=4.15$. Results of a convergence study with varying $N_{1 / 2}$, presented in Arienti (2002), show that the main wave features are essentially converged in these examples when $N_{1 / 2}=22.5$.

The height of the computational domain (top-to-bottom) is approximately $250 \Delta_{1 / 2}$. The time step is $1.1 \times 10^{-3}$, corresponding to an average CFL number of 0.5 or smaller; given a final time between 40 and 60 for the detonation front to reach the bottom of the computational domain, at least 40000 time steps are required to reach simulation completion.

\subsection{Case $\theta_{C J}=1$}

Two numerical schlieren images are displayed in figure 7. Numerical schlieren visualization amounts to displaying the magnitude of the density gradient as a grey-scale. A nonlinear mapping, or grey-scale shading function, is used so that density gradients 
(a)

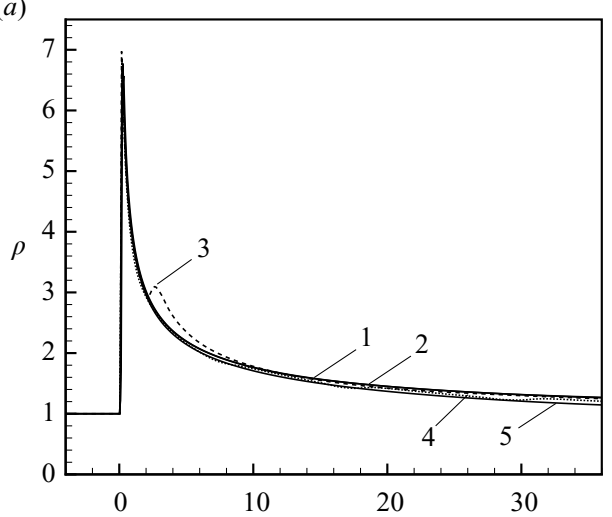

(c)

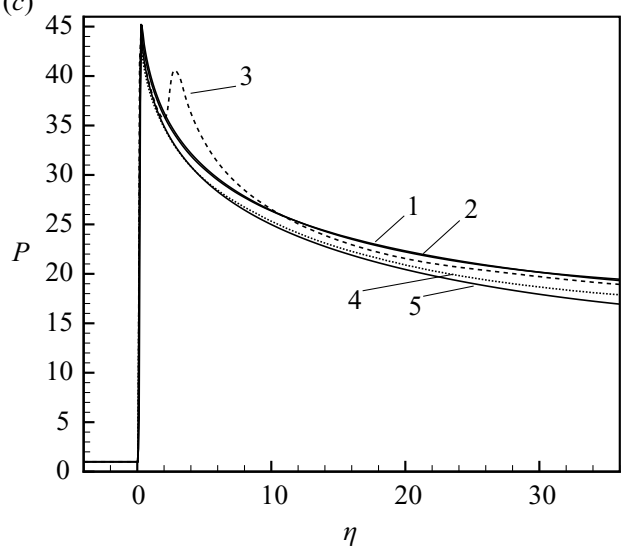

(b)

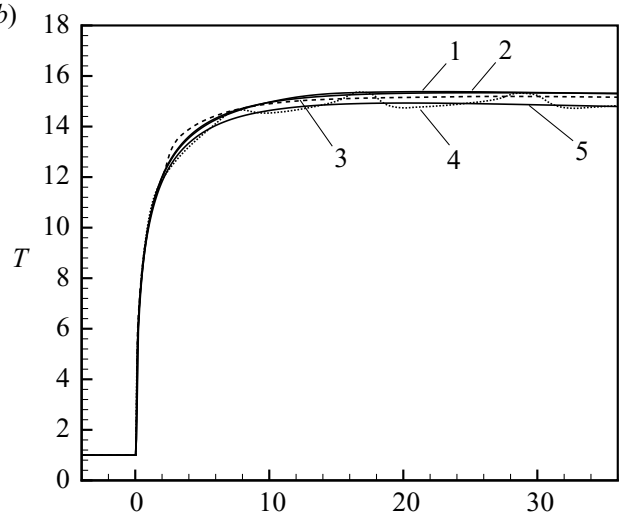

(d)

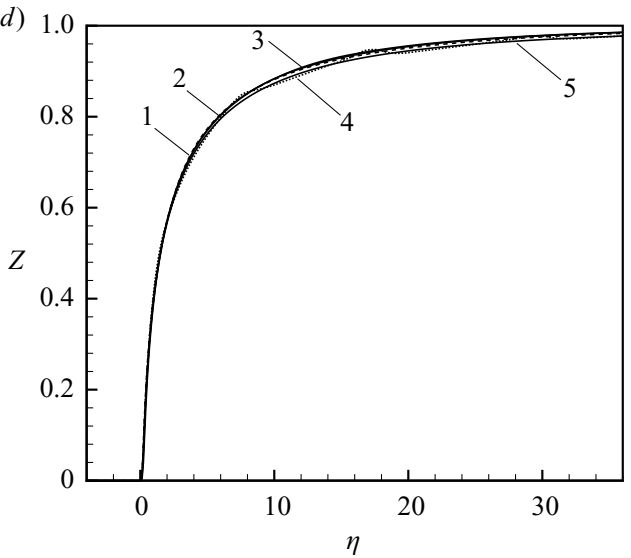

Figure 8. $(a)$ Density, $(b)$ temperature, $(c)$ pressure and $(d)$ progress variable profiles for 5 data sets extracted at $t=35.79$. Slices 1 and 5 are extracted along the plane of symmetry and the corner wall, respectively. The remaining data are taken in the shock normal direction and are evenly spaced along the detonation front.

varying through several orders of magnitude are still visible. In this work, the greyscale shading function is

$$
v=0.8 \exp \left(-\mu \frac{|\nabla \rho|}{|\nabla \rho|_{\max }}\right),
$$

with $\mu$ a strictly positive amplification parameter. The grey-scale ranges from black for $\nu=0$ to white for $\nu=1$. Larger values of $\mu$ give darker images and accentuate weak features of the flow. To provide a consistent grey-scale reference, frames in a sequence of schlieren images, such as that in figure 7 , have the same amplification and normalization factors.

In addition to the density gradient, figure 7 also displays the locus of points where the product mass fraction is equal to 0.95 . This contour is displayed as a solid line. The corner, on the left-hand side of each plot, is shown as a rectangular shape with a small (not visible to the eye) rounded vertex of radius $r_{c}=1$. In each frame, only the last portion of the inlet channel (one-fifth of the total length) is shown.

As mentioned in the previous section, a value $\theta_{C J}=1$ corresponds to a reaction rate model that is essentially insensitive to the shock velocity changes in the expansion from the channel half-width $H=36.67$. Figure 8 is a plot of density, temperature, 
(a)

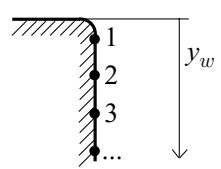

(b)

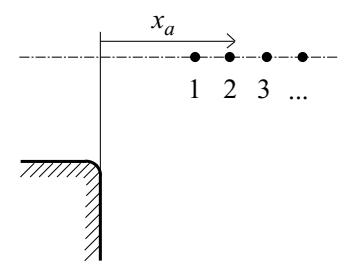

FIGURE 9. (a) Initial location of injected particles along the corner wall and the $(b)$ plane of symmetry.

(a)

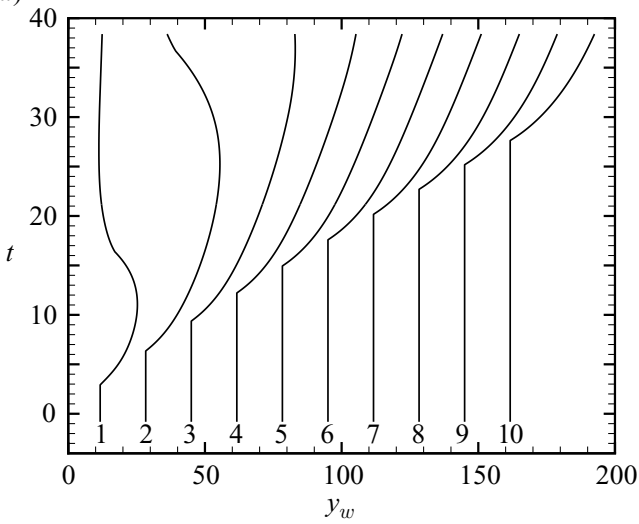

(b)

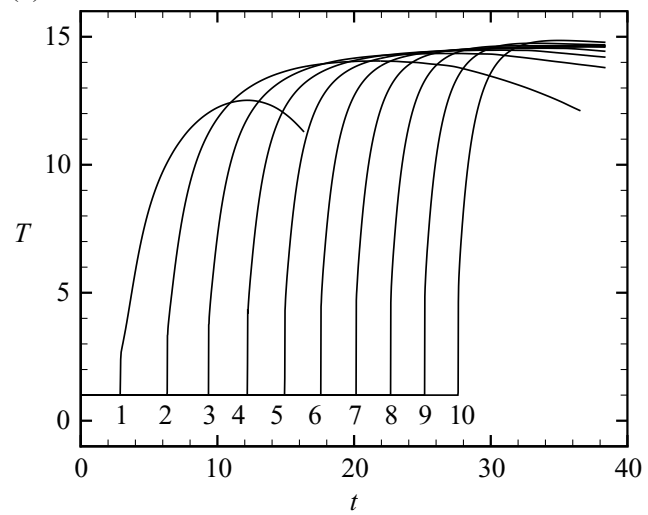

Figure 10. (a) Particle paths for 10 sample particles injected along the vertical corner wall for $\theta_{C J}=1$. $(b)$ Temperature profiles along the particle paths.

pressure and progress variable for five 'slices' of the computational domain at time $t=35.79$. Slices 1 and 5 are extracted along the plane of symmetry and the corner wall, respectively. The remaining data are taken in the shock normal direction and are evenly spaced along the detonation front (see figure $7 a$ ). In all the slices, the postshock pressure is almost exactly $75 \%$ of the von Neumann value, corresponding to a detonation velocity $0.86 D_{C J}=7.010$. This estimate is consistent with the diagram in figure 4. Overall, the profiles in the two frames show the same dependence on $\eta$, with the exceptions of slice 3 (dashed line) and slice 4 (dotted line) both passing through a system of transverse shocks. No residual effects of the transverse gradient due to the corner rarefaction are observed, and, by the shock-change equation (Fickett \& Davis 1979, p. 101), we conclude that the front is propagating as an almost cylindrical detonation.

The receding 0.95 reaction locus in the first frame of figure 7 indicates that the most severe reduction of reaction rate is found along the corner wall. The trajectories of particles injected along this boundary (see figure $9 a$ ) are displayed in the spacetime diagram of figure $10(a)$. The temperature profiles in figure 10(b) show that all particles ignite, even those very close to the corner. From their trajectories, it appears that these particles are eventually pulled upward into the colder fluid at the corner vortex. At this time, however, the fluid element has almost completely reacted, and the decrease in temperature has no feedback to the main reaction zone.

We conclude the analysis of the case $\theta_{C J}=1$ with a comment on the group of transverse shocks shown in figure $7(b)$. The first of these waves, moving from the corner to the centre of the channel, is due to reflection of the curved detonation 
(a)

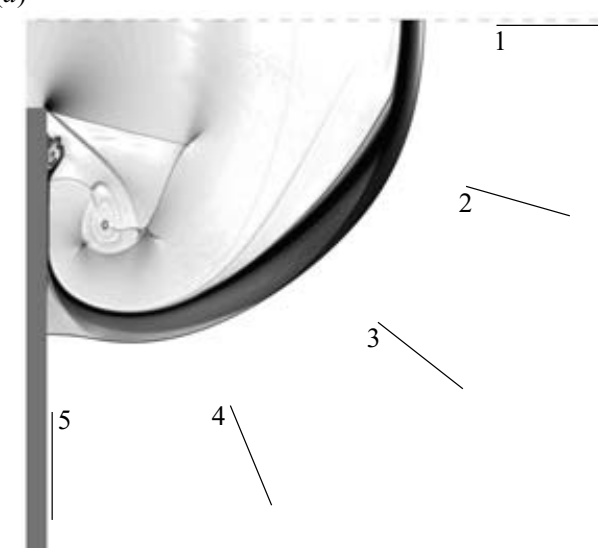

(b)

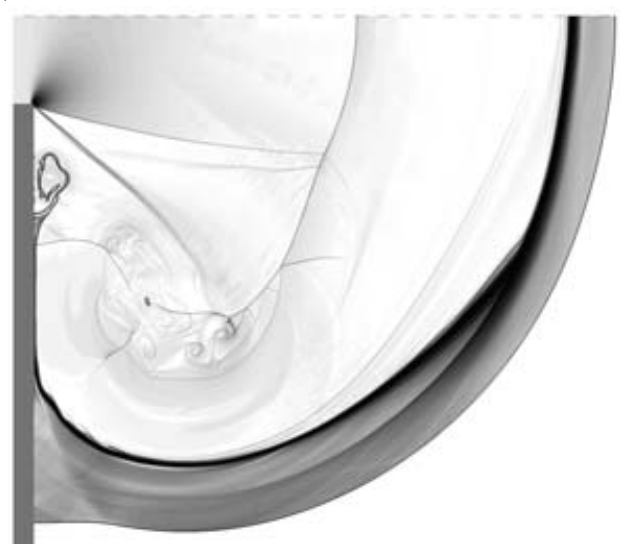

FIGURE 11. Numerical schlieren images for the case $\theta_{C J}=4.15$ at $(a) t=28.43 ;$ (b) 53.22.

front at the corner wall in the initial phase of corner turning. The reflection causes a triple point to form at the detonation front with a contact discontinuity embedded in the reaction zone. We found that the discontinuity in the sonic parameter acts as a channel for the propagation of high-frequency acoustic disturbances, which are amplified by the energy release due to chemical reaction and steepen to form new transverse shocks. To each new shock at the detonation front corresponds a contact discontinuity, which, in turn, acts as a propagation guide. The mechanism described here is consistent with the propagation and amplification of high-frequency acoustic waves in a planar ZND-CJ reaction zone described by Strehlow \& Fernandes (1965) and Barthel \& Strehlow (1966). A complete discussion on this topic can be found in Arienti (2002).

\subsection{Case $\theta_{C J}=4.15$}

Two snapshots of the simulation, computed for $\theta_{C J}=4.15$, are displayed in figure 11 . The decoupling occurs just behind the head of the corner signal that sweeps across the wave from the corner to the centre of the channel. The detonation fails completely and there is no local re-ignition, so the main flow features appear to evolve in time, similarly to a non-reacting shock.

Moving along the wavefront from the channel centre, we see that the wave curvature increases up to the point where the no-flow boundary condition, generated by the wall, causes a straight shock perpendicular to it. The transition from curved to straight front is smooth, since the shock is immediately followed by an unsteady expansion wave. This structure is qualitatively predicted by Whitham's geometrical shock dynamics applied to a non-reacting shock diffracting at a sharp corner (Whitham 1974, p. 297). It is also observed in direct numerical simulations by Xu, Aslam \& Stewart (1997) and Helzel, Leveque \& Warnecke (2000). At the junction of the curved front and the stem adjacent to the wall, the schlieren images in figure 11 indicate a discontinuous change of density gradient.

The steep density gradient in figure 11 marks the separation between the burnt gases, produced before reaction quenching, and the shock-compressed (but unburnt) reactants. This is shown in figure 12 , where density, temperature, pressure and progress variable profiles of five slices of the computational domain (at time $t=53.22$ ) are plotted. Slices 1 and 5 are extracted along the plane of symmetry and the corner wall, 
(a)

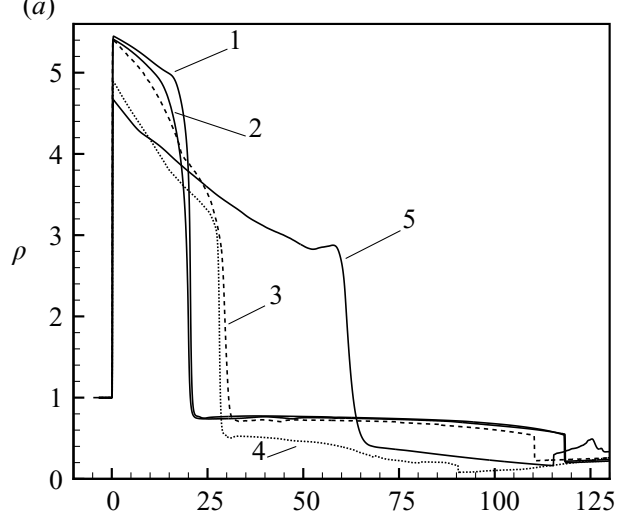

(c)

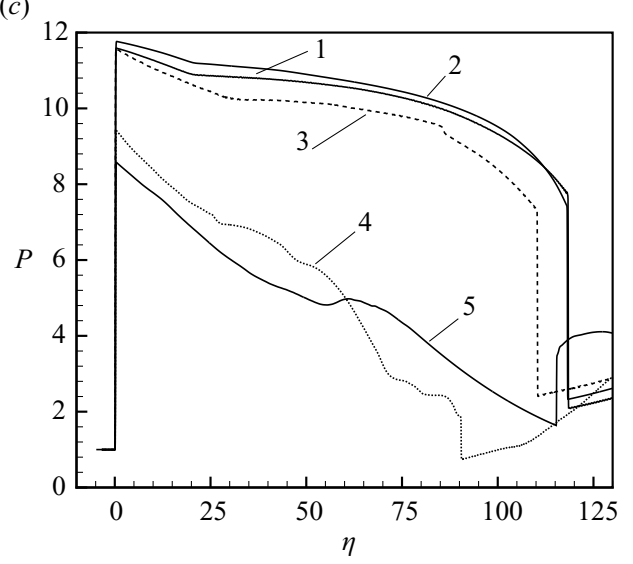

(b)

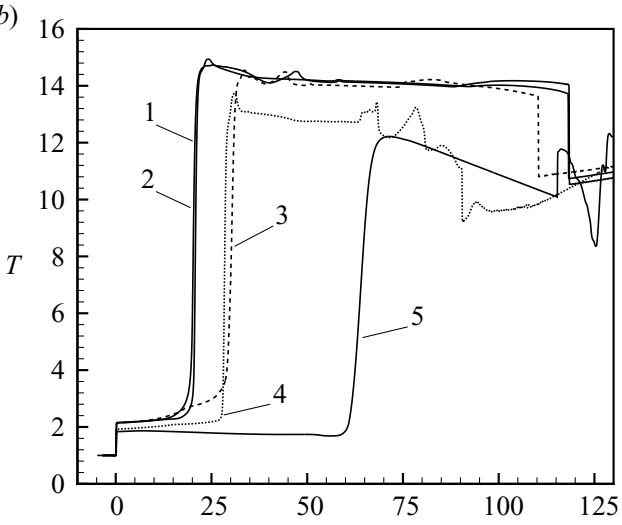

(d)

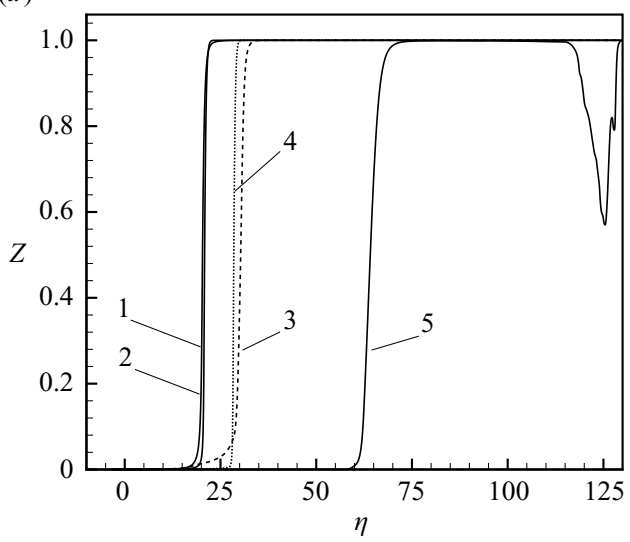

Figure 12. $(a)$ Density, $(b)$ temperature, $(c)$ pressure and $(d)$ progress variable profiles for 5 data sets extracted at $t=53.22$.

respectively. The remaining data are taken in the direction normal to the shock and are evenly spaced along the detonation front (see figure 11a). Behind the lead shock, temperature and progress variable do not vary substantially from their post-shock values, whereas density and pressure decrease similarly to what would be expected in a blast wave. Density drops abruptly at a distance of 20 to $30 \Delta_{1 / 2}$ from the shock. At the same location, temperature and progress variable rise very rapidly to the corresponding values of the burnt products. This distance becomes $60 \Delta_{1 / 2}$ behind the Mach stem at the wall (slice 5).

\subsubsection{Particle analysis}

We now discuss the results obtained for a selection of particles injected along the channel plane of symmetry (figure $9 b$ ). That is the most convenient location to probe the flow field, where the corner expansion is initially the weakest. For each particle, data analysis starts immediately after the passage of the lead shock. Since the shock is normal to the plane of symmetry, the simplified equation (2.13) can be used. Particle trajectories, labelled from 1 to 10 in figure 13(a), become almost parallel to the traces of the constant mass fraction of the product. This is an indication that the reaction is quenched, with no change in reactant-product composition (see also the contact discontinuity in figure 11).The temperature history along particle trajectories is plotted 
(a)

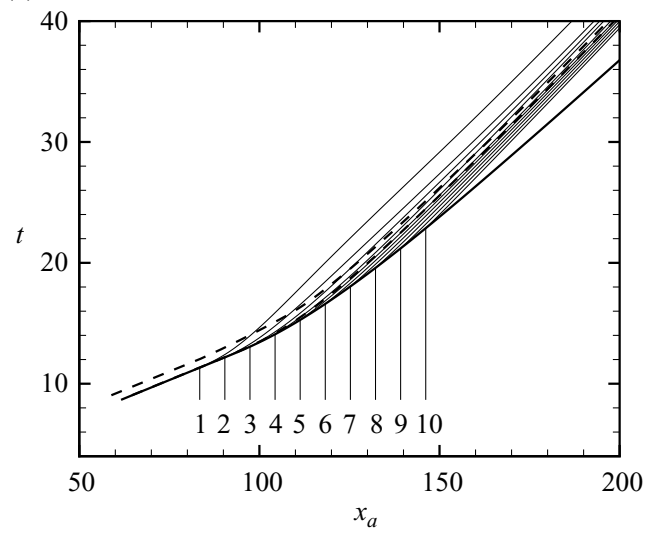

(b)

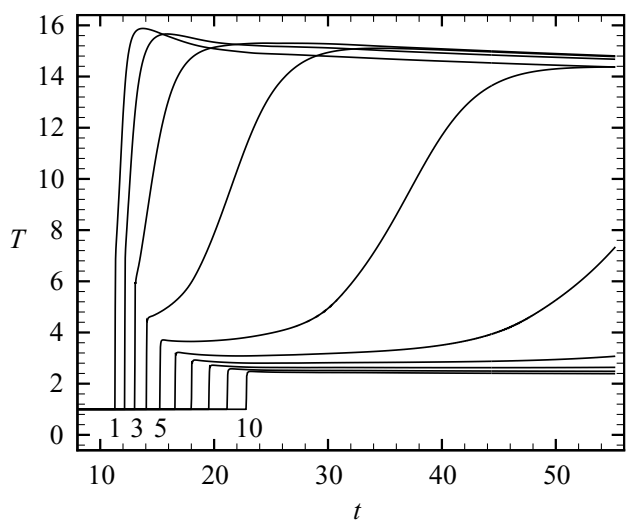

Figure 13. (a) Particle paths for ten sample particles on the plane of symmetry. Shock (thick solid line); traces of $5 \%$ and $95 \%$ of reaction completion (dashed lines); particle paths (thin solid lines). (b) Temperature profiles along the particles paths.

in figure 13(b). The increase of the particle's time to ignition as the shock strength decreases is clearly consistent with the reaction rate dependence on temperature.

The decomposition of $\mathrm{D} T / \mathrm{D} t$ is performed for particles 1, 3,5 and 10 and displayed in figure 14. The plots show that the (positive) transverse divergence term is always small. This result, also found in the analysis of the $\theta_{C J}=1$ and $\theta_{C J}=3.5$ cases, indicates that near the plane of symmetry, the flow is nearly cylindrical. From figure 14, we also note that the contribution made by the curvature term to $\mathrm{D} T / \mathrm{D} t$ is negligible for particles close to failure.

The unsteady term mainly balances curvature and transverse divergence in figure 14(a), while the temperature variation is produced by heat release alone. Plots similar to figure $14(a)$ are obtained for the case $\theta_{C J}=1$. In figures $14(b)$ and $14(c)$, the unsteady term becomes a large negative forcing factor that reduces DT/D $t$ below the value due to heat release for particles 3 and 5 , whose rise in temperature is well separated from the initial shock. For particle 5, in particular, unsteadiness is initially larger than heat release, so that immediately after the shock, the temperature decreases instead of increasing. The large delay in ignition of particle 5 is not found for the case $\theta_{C J}=1$. This behaviour is more and more evident in the following elements of fluid, until, by particle 10, the temperature steadily decreases after the shock, owing to unsteadiness dominating over heat release (figure $14 d$ ). At this point, the reaction is completely quenched.

\subsection{Case $\theta_{C J}=3.5$}

This example shows the most interesting behaviour of all the cases examined in this study: the detonation begins to fail at the wall, but, at some point, a re-ignition event occurs. Numerical schlieren images (figures 15 and 16) for the case $\theta_{C J}=3.5$ indicate how complex the dynamics of the diffraction process are. As shown by figures 4 and 5 , the front evolves differently along the plane of symmetry and the wall. At the channel centre, the shock appears never to completely decouple from the reaction zone. The detonation speed exhibits a plateau at a speed of about $0.6 D_{C J}$, but then climbs toward the $\mathrm{CJ}$ value. Conversely, the shock at the corner wall immediately detaches from the reaction zone and maintains a speed of about $0.4 D_{C J}$ until the arrival of a re-ignition transverse wave. 
(a)

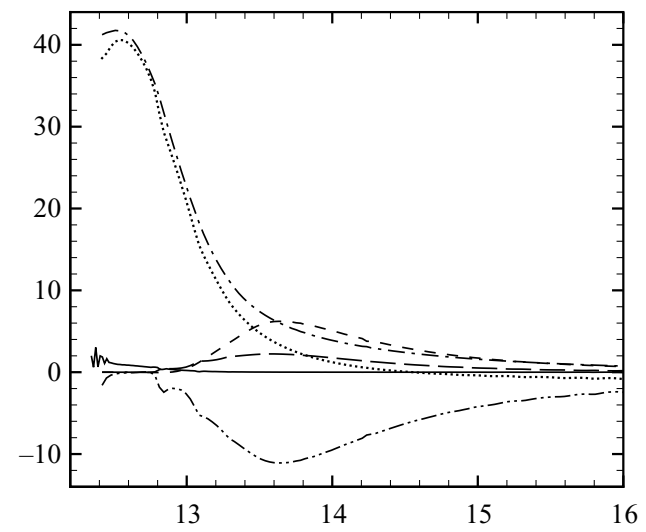

(c)

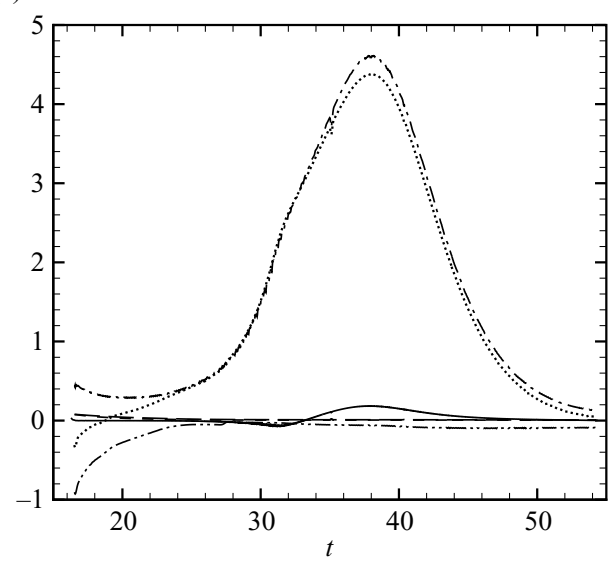

(b)

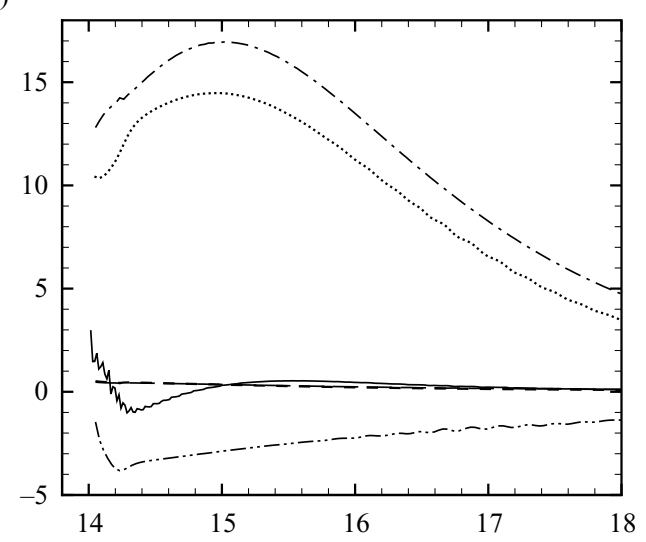

(d)

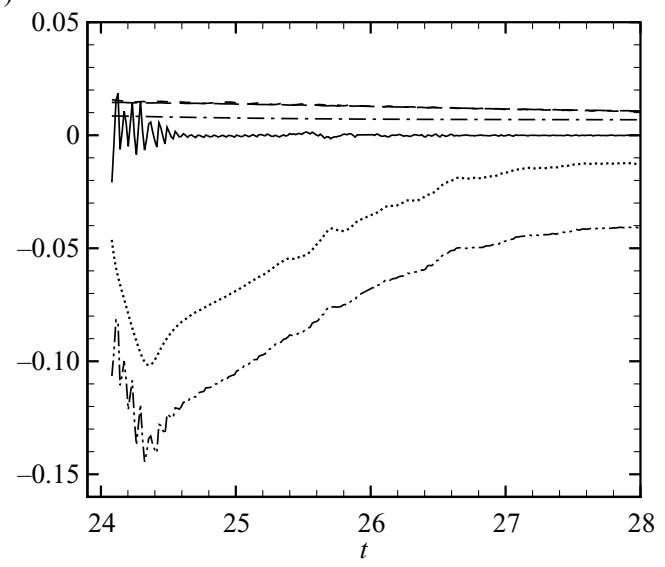

FIGURE 14. Terms in the reaction zone temperature (2.13) along the same particle paths as in figure 13 for $\theta_{C J}=4.15$. The particles are injected on the channel plane of symmetry. $\cdots$, Lagrangian temperature; $-\cdot-$, heat release; --- , curvature; -- , transverse divergence; $-\cdot-$, unsteadiness. The solid line is the difference between the left-hand side and the right-hand side in (2.13), as computed from the terms above. (a) Particle 1; (b) Particle 3; (c) Particle 5; (d) Particle 10.

From figure $15(a)$ to figure $15(c)$, the rarefaction originating at the corner reflects at the plane of symmetry, and then sweeps downwards along the detonation front. This further reduces the strength of the lead shock and results in a flattening of the front at the centre of the channel. An observer moving along the wavefront from the channel centre to the corner wall would find the maximum value of pressure at the lead shock to be immediately ahead of the reflected expansion front. As the observer moves beyond that peak toward the corner wall, the pressure decreases again, this time because of the effect of the first corner expansion. At time $t=17.49$, the reflected expansion appears to have lost most of its strength and, near the wavefront pressure peak, the end reaction zone begins to approach the shock, which accelerates. At time $t=21.15$, the wavefront has acquired a very peculiar shape. It is almost flat at the plane of symmetry, has a relatively large curvature in the centre, and is completely decoupled at the wall. A fold or kink in the shock front is starting to form. In the next frame, a transverse shock wave is developing from this fold and propagating 
(a)

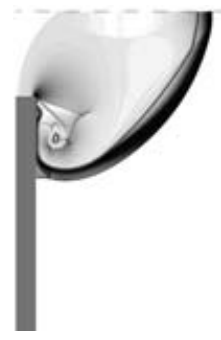

(d)

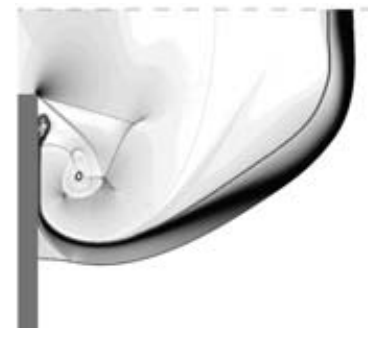

(b)

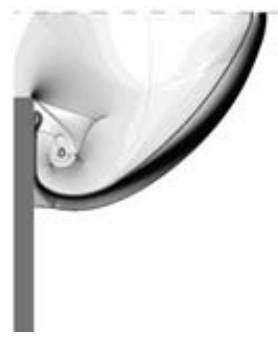

(e)

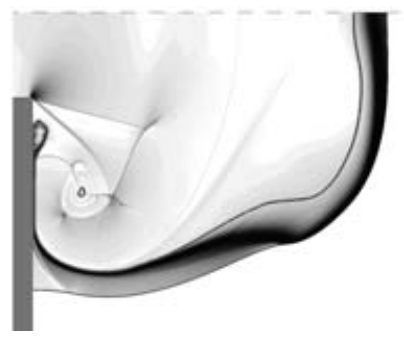

(c)

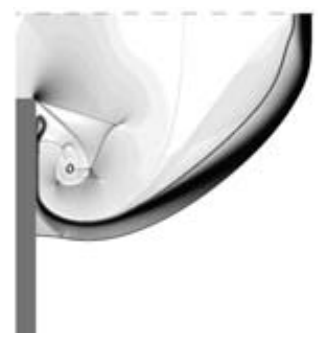

(f)

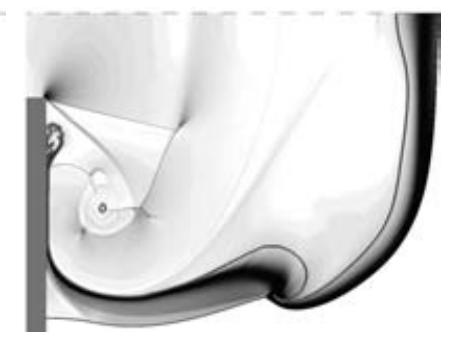

FIGURE 15. Numerical schlieren images for the case $\theta_{C J}=3.5$. (a) 10.17 ; (b) 13.83 ; (c) 17.49; (d) $21.15 ;(e) 24.81 ;(f) 28.47$. The solid line is the locus of $95 \%$ product.

toward the corner wall. Figure 16 displays this further evolution, up to the reflection of the transverse wave at the corner wall.

A close-up of the transverse wave system at $t=28.47$ is shown in figure 17 . The incident shock (IS) is essentially non-reactive, while the curved Mach stem (MS) has a much higher reaction rate. This can be seen by considering the distance of the $95 \%$ reaction locus (solid line in figure $17 b$ ) from the shock front. A contact discontinuity (CD2) separates the partially reacted gas, processed by IS, from the completely burnt products. The transverse wave (TS) extends between the two triple points, T1 and T2, as a straight shock, and from T2 to the $95 \%$ reaction locus as a curved strongly reactive wave. It propagates into the partially reacted region behind the incident shock and quickly brings the reaction to completion. The maximum value of reactivity is found immediately behind the short stem connecting point $P$ with T2. In figure 17 $(a)$, this small area is above the cutoff value of the pressure contours, and it is surrounded by the highest density of contour lines. The contact discontinuity CD1 separates the gas that has passed through the MS from the gas processed by the transverse shock. A relatively minor feature, a kink $(\mathrm{K})$ in the Mach stem, is also visible. The discontinuity associated with the kink terminates near point $P$. Grid resolution studies by Sharpe (2001) indicate that this feature rapidly disappears in under-resolved simulations, so its presence in the result we are showing here suggests that the level of resolution is adequate.

It is important to point out that a transverse wave structure analogous to that described here can also be observed with higher values of normalized activation energy. In the case computed with $\theta_{C J}=3.75$ (not shown here), the mechanism described above was found to produce a first transverse wave similar to that in figure 17. This wave was slower and weaker than that at $\theta_{C J}=3.5$ and could not accelerate the lead shock. This is shown in figure 4 , where $D_{a}$ decays monotonically for $\theta_{C J}=3.75$. It is, therefore, the acceleration of the shock close to the channel 
(a)

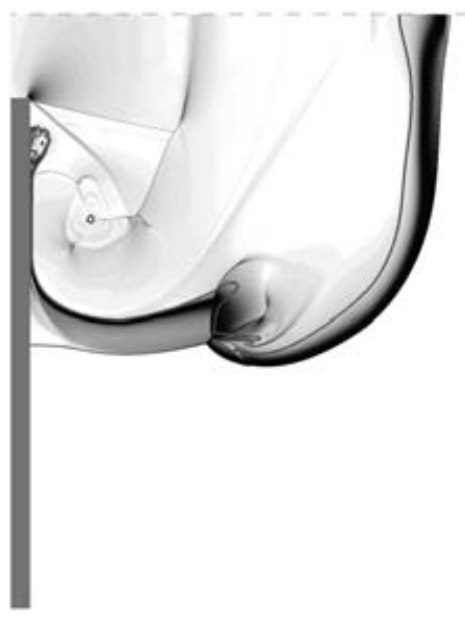

(c)

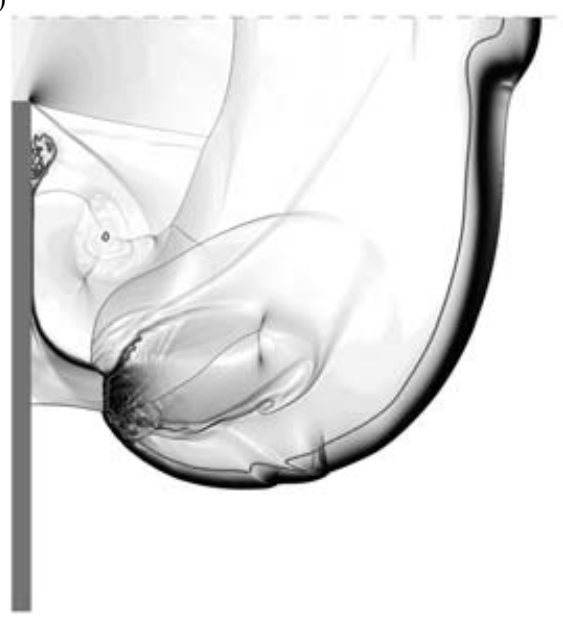

(e)

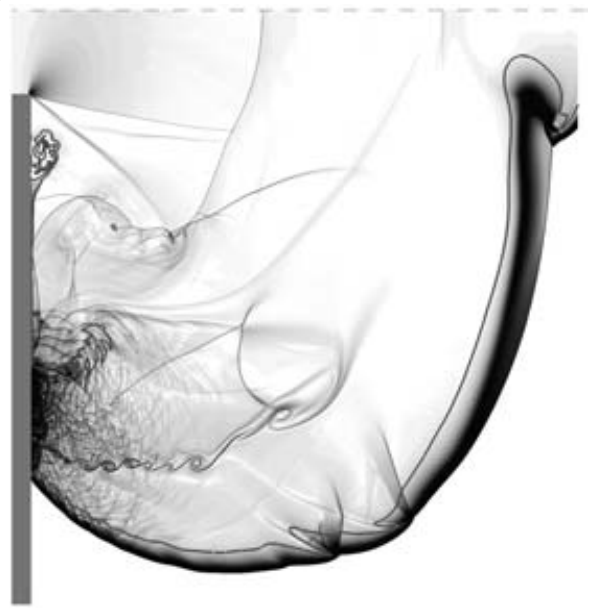

(b)

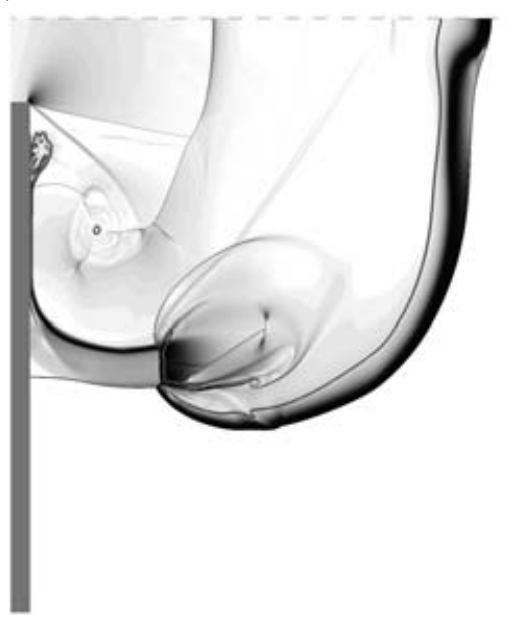

(d)

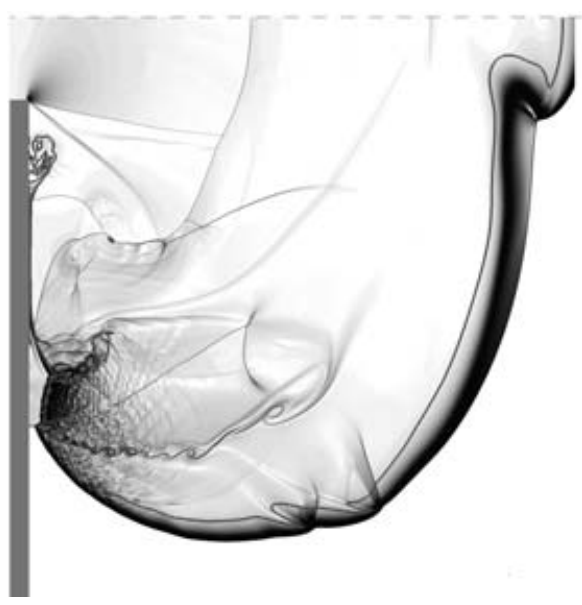

(f)

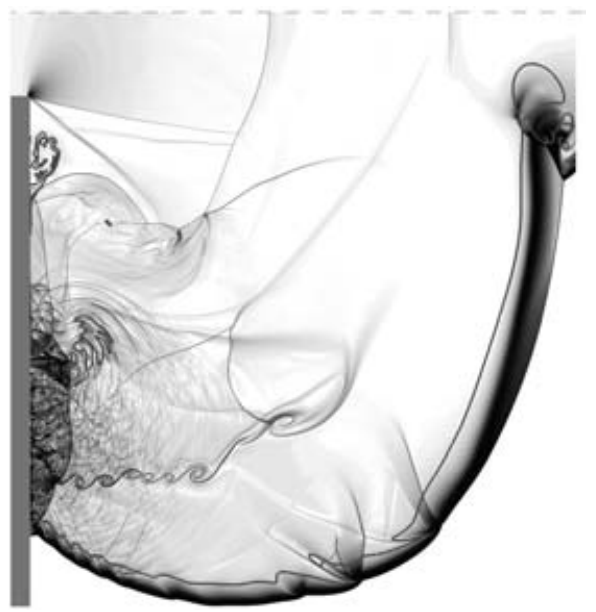

FiguRE 16. Numerical schlieren images for the case $\theta_{C J}=3.5$. (a) 32.13 ; (b) 35.79 ; (c) 39.45 ; (d) 43.11; (e) 44.94; $(f) 46.77$. 
(a)

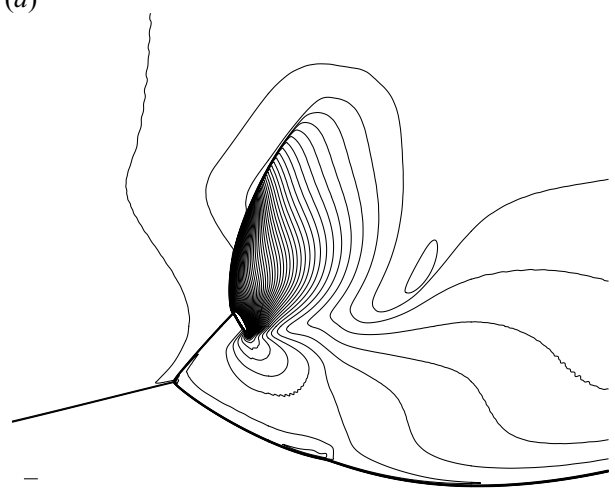

(b)

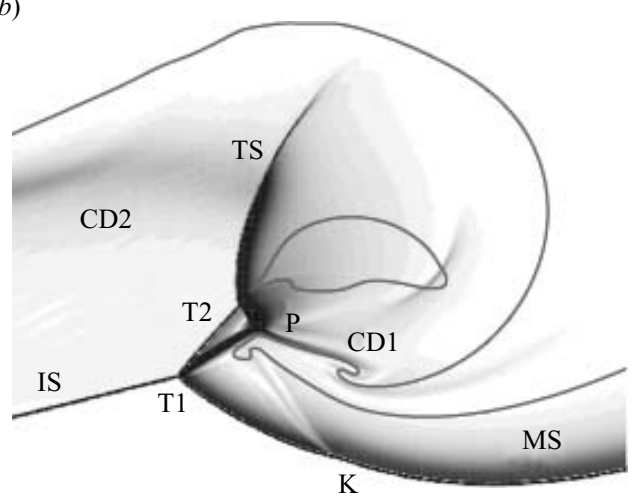

FIGURE 17. Structure of the re-ignition transverse wave: $(a)$ contours of pressure and (b) numerical schlieren of density, at time $t=28.47$. In $(a)$, the contour lines are spaced by the non-dimensional value 2.083 with a cutoff limit of 250 marking the pressure peak (at 445) behind the kink. The segment at the bottom left shows the length $\Delta_{1 / 2}$ in the plot scale. The solid line in $(b)$ is the $95 \%$ reaction completion locus.

(a)

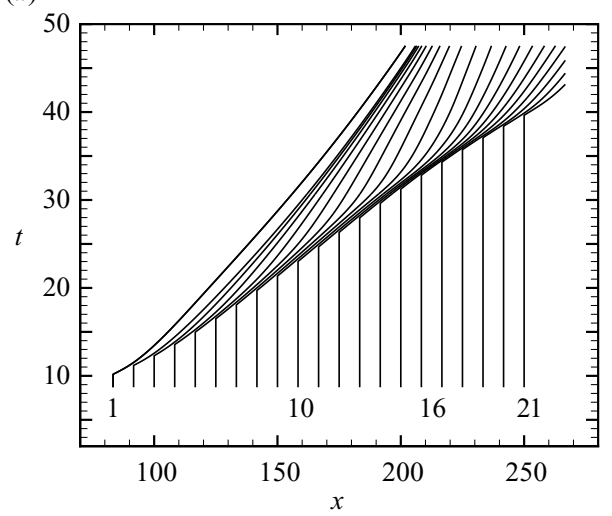

(b)

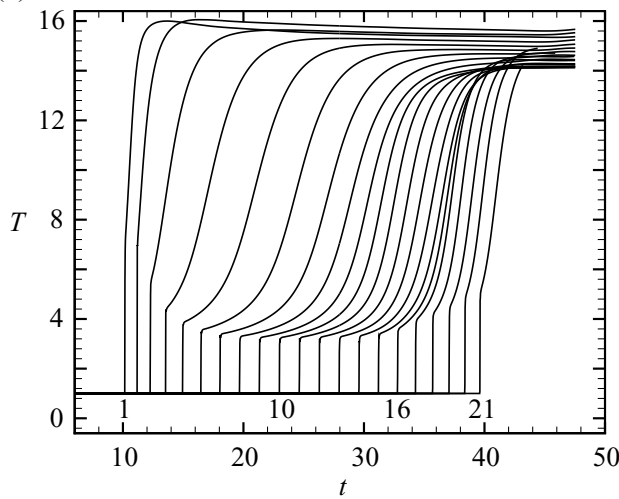

Figure 18. (a) Particle paths for 21 sample particles injected along the channel plane of symmetry (figure $9 b$ ) for $\theta_{C J}=3.5$. The labels $1,10,16,21$ indicate particles that are analysed in terms of numerical dominant balance. $(b)$ Temperature profiles along the particle paths.

plane of symmetry that appears as the distinctive feature of a successful near-critical detonation diffraction. This point will be clarified in the following sections.

\subsubsection{Particle analysis}

From the previous description, two radically different behaviours can be identified in the two regions near the channel plane of symmetry and the corner wall. At the wall, the decoupling of the shock from the reaction zone persists until an external cause, the reflection of a strong transverse shock, is able to re-ignite the mixture. Near the channel centre, a complex wavefront dynamic results in shock folding and eventually produces an explosion, followed by a system of transverse waves. In both cases, local flow symmetry occurs, and the simplified equation (2.13) for the Lagrangian temperature derivative can be used.

Lagrangian trajectories are displayed in figure 18(a) for particles that are located on the plane of symmetry and in figure $19(a)$ for particles on the wall. The temperature 
(a)

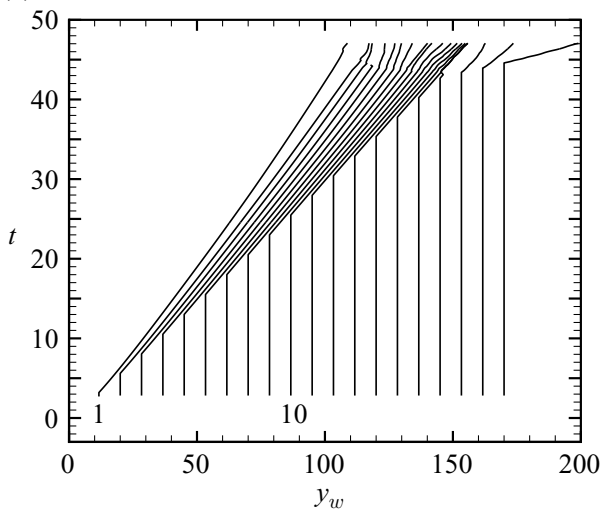

(b)

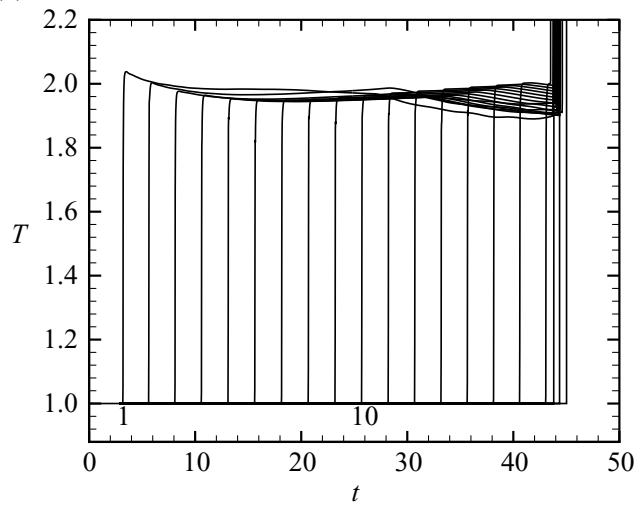

FIgURE 19. (a) Particle paths for 20 sample particles injected along the vertical corner wall (figure $9 a$ ) for $\theta_{C J}=3.5$. The labels 1 and 10 indicate the particles that are analysed in terms of numerical dominant balance. (b) Temperature profiles along particles paths.

readings along these paths are displayed in figures $18(b)$ and $19(b)$, respectively. Labels indicate particles that will be further analysed in this section.

For particles moving along the channel plane of symmetry, the post-shock temperature decreases, reaches a minimum, and then increases again. Likewise, the time delay between the shock passage and the peak temperature for particle 10 is larger by about an order of magnitude than the corresponding time for particle 1 , indicating a growth in ignition time. From particles 10 to 21, the ignition time decreases again. These results indicate that the detonation slows down, but does not fail. Note that the post-shock slopes of the temperature never become negative for any of the trajectories shown in figure 18. This important observation, and the fact that $\mathrm{D} T / \mathrm{D} t$ instead passes through zero when $\theta_{C J}=4.15$, will be used later in the discussion section.

In particles moving along the corner wall, no ignition occurs until approximately $t=43$. The post-shock temperature steadily decays from particle 1 to particle 7 , has a slight increment from particle 7 to 12 , and then decreases again. Overall, the flow field near the wall can be treated as non-reacting up to the point when ignition is suddenly re-started by the transverse shock reflection (between figures $16 d$ and $16 e$ ).

The decomposition of the terms in the reaction zone temperature according to (2.13) is shown in figure 20 for particles $1,10,16$ and 21 along the plane of symmetry of the channel. Results for particles initially located just downstream of the head disturbance arrival point are very similar for the cases $\theta_{C J}=3.5$ and $\theta_{C J}=4.15$ (see figure $20 a$ and figure $14 a$ ). This is expected since the activation energies in these two cases differ by a relatively small amount. The behaviour of the particles that follow is, however, radically different. Whereas particles 10 and 16 have much longer ignition times, of the order of 5-10 units of time, particles that are located further downstream again display an ignition time close to one. This reinforces the observation that for the case $\theta_{C J}=3.5$, the detonation re-couples near the channel centre, whereas for the case $\theta_{C J}=4.15$, the detonation completely decouples.

If we now examine one by one the terms appearing on the right-hand side of (2.13), we notice that the unsteady term appears almost everywhere in the form of a negative forcing factor, as for the case $\theta_{C J}=4.15$. Overall, curvature and transverse divergence effects play a relatively unimportant role in determining the Lagrangian derivative of 
(a)

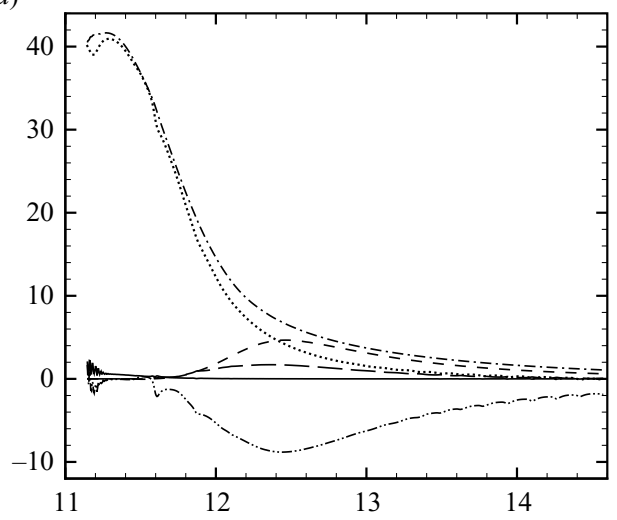

(c)

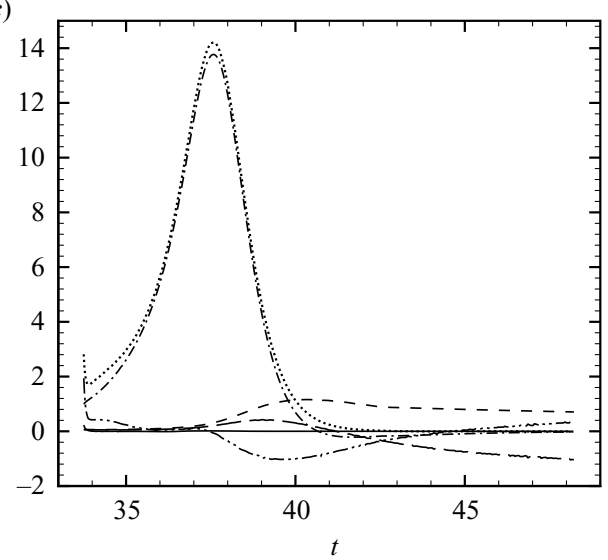

(b)

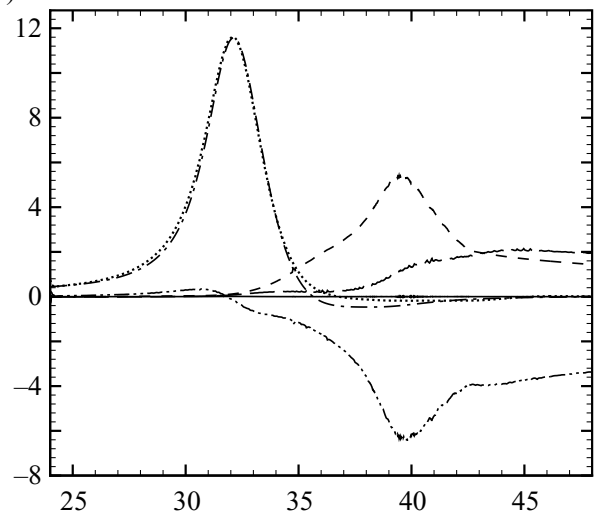

(d)

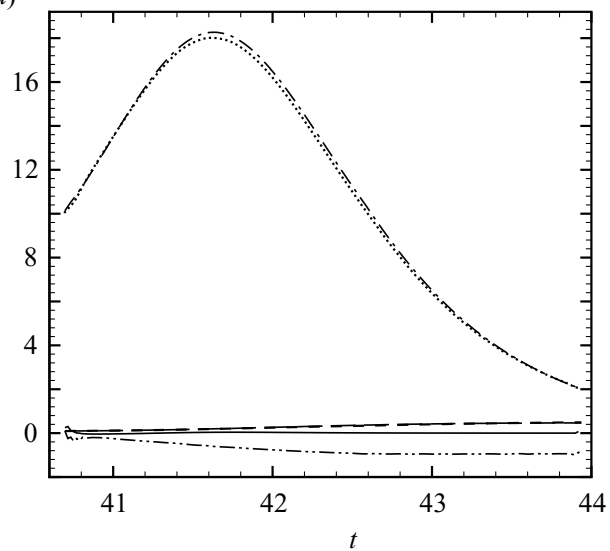

FiguRE 20. Terms in the reaction zone temperature (2.13) along the same particle paths as in figure $18(a)$ for the case $\theta_{C J}=3.5$. The particles are injected along the plane of symmetry...., Lagrangian temperature; $-\cdot-$, heat release $;---$, curvature; -- , transverse divergence; $-\cdot \cdot-$, unsteadiness. The solid line is the difference between the left-hand side and the right-hand side in (2.13), as computed from the terms above. (a) Particle 1; (b) Particle 10; (c) Particle 16; (d) Particle 21.

temperature. Exceptions to this are the paths 10 and 16 (figure 20b,c), where a strong peak in the curvature term can be observed at time $t \cong 40$. At this point, however, the flow has already reacted, and $\mathrm{D} T / \mathrm{D} t$ is almost zero.

The analysis of fluid elements along the corner wall (not shown here) leads to results that are very similar to those observed for the case $\theta_{C J}=4.15$, with delayed or no ignition occurring and temperature decrease dictated by the unsteady term. Temperature variations are much smaller than those observed in particles moving along the plane of symmetry until the arrival of the re-ignition transverse wave at approximately $t=43$. Further analysis after this point is not possible owing to the presence of strong shock waves processing the unreacted fluid.

\section{Discussion}

In the previous section, the magnitude of the terms in the reaction zone structure equation was examined along selected particle paths for sub-, near- and super-critical 
cases. The study focused on particle trajectories running along the plane of symmetry of the channel and the lower wall of the corner, since these two regions show very different wavefront dynamics in the case $\theta_{C J}=3.5$.

A first result of our analysis is that the contribution of the curvature and transverse divergence terms to the Lagrangian derivative of temperature is negligible in causing particle ignition delay. This result coincides with the findings of Eckett et al. (2000) for the closely related case of direct initiation of spherical detonations. Thus, the reaction zone structure equation simplifies to show the competition between energy release rate and unsteadiness,

$$
\begin{aligned}
\left(1-M^{2}\right) C_{p} \frac{\mathrm{D} T}{\mathrm{D} t}=\left(1-\gamma M^{2}\right) Q k \rho(1-Z) \exp ( & \left.\frac{-E_{a}}{T}\right) \\
& +w_{\eta}\left(D_{a}-w_{\eta}\right)_{, t}+\frac{P_{, t}}{\rho} .
\end{aligned}
$$

A second result is that fluid elements that fail to ignite exhibit a negative post-shock derivative $\mathrm{D} T / \mathrm{D} t$. Two representative cases were shown in the previous section. In the case $\theta_{C J}=4.15$, the derivative $\mathrm{D} T / \mathrm{d} t$ is negative along several Lagrangian paths after the passage of the shock (figure 13), but when $\theta_{C J}=3.5$, the derivative remains positive at post-shock conditions, albeit very close to zero for a few particles (figure 18). To associate the temperature change immediately after the passage of the lead shock to the particle ignition delay, we propose the local decoupling condition,

$$
\mathrm{D} T /\left.\mathrm{D} t\right|_{s}=0,
$$

as a criterion for the decoupling of the shock front from the reaction zone. Equation (5.2), evaluated at the post-shock state, $s$, intuitively relates the rate of post-shock temperature increase to the ignition delay of the particle. To satisfy this condition introduced by Eckett et al. (2000) in the study of direct detonation initiation and postulated by Schultz (2000) for detonation diffraction - the following equation must be satisfied,

$$
Q k \rho_{s} \exp \left(\frac{-E_{a}}{T_{s}}\right)=-\frac{1}{\left(1-\gamma M_{s}^{2}\right)}\left[w_{\eta}\left(D_{n}-w_{\eta}\right)_{, t}+\frac{P_{, t}}{\rho}\right]_{s} .
$$

Note that the right-hand side of (5.3) is a function only of $\gamma$ and the shock deceleration.

A third result is that the global criterion for diffraction failure can be reduced to a test for detonation front decoupling at the plane of symmetry of the channel, a few reference reaction lengths downstream of the head expansion arrival point. We call this location the critical point. The fact that re-ignition starts from the channel centre has been regularly observed in experimental studies on detonation diffraction (see, for instance, Edwards et al. 1979; Moen et al. 1982).

In the strong shock limit, equation (5.3), evaluated at the plane of symmetry, becomes

$$
Q k \exp \left(-\frac{D_{C J}^{2}}{D_{a_{c}}^{2}} \theta_{C J}\right)=-\frac{12}{3-\gamma} \frac{(\gamma-1)^{2}}{(\gamma+1)^{3}} D_{a_{c}} \dot{D}_{a_{c}} .
$$

Equation (5.4) points out the balance between the energy release rate, proportional to $Q k$, and a term similar to kinetic energy decay. To characterize the critical detonation 


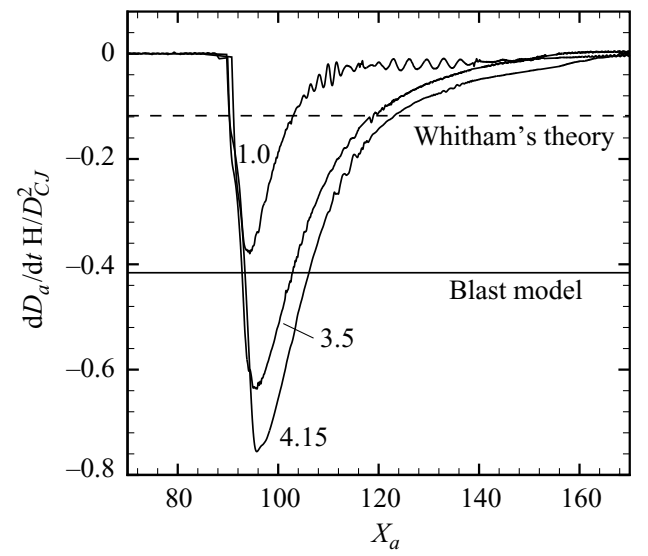

FIGURE 21. Shock deceleration as a function of the distance from the corner vertex, parameterized by $\theta_{C J}$.

speed $D_{a_{c}}$, we take (somewhat arbitrarily) the critical point to be such that

$$
D_{a_{c}}=0.75 D_{C J},
$$

and propose that, if (5.4) is verified downstream of this point, the decoupling will continue until reaction quenching. It is very difficult to relate $D_{a_{c}}$ to the dynamic and kinetic properties of the decoupling detonation front, to the point that this term should be considered more like a free parameter of the model. The same factor 0.75 is derived by Radulescu et al. (2003) from experiments on direct detonation initiation as the critical value marking the onset of detonation.

To derive the critical relation between $\theta_{C J}$ and $H$ from (5.4), we must still model the critical shock deceleration at the channel centre, $\dot{D}_{a_{c}}$. Shock deceleration depends on the initial speed and the width of the channel, and it can be argued to be proportional to $D_{C J}^{2} / H$ by dimensional arguments. By assuming that, in the sub-critical case, the lead shock decays (at the plane of symmetry) as a cylindrical blast of initial radius $H / \tan \alpha$, the relation between shock speed and curvature is $D_{a_{c}}=\kappa H D_{C J} / \tan \alpha$. The shock decay at $H / \tan \alpha$ is, therefore,

$$
\dot{D}_{a_{c}}=-\frac{D_{C J}^{2}}{H} \tan \alpha .
$$

The angle $\alpha$ is the maximum disturbance angle from the Skews' construction (see figure $1 b$ ). For this problem, $\alpha=22.6^{\circ}$. The shock decay computed from the numerical simulations is plotted in figure 21 as a function of position for the three values of $\theta_{C J}$, together with the estimated critical shock deceleration deduced from the blast model. An alternative formula for $\dot{D}_{a_{c}}$ can be derived from Whitham's geometrical shock dynamics (see Arienti 2002 for details) and is also shown in figure 21.

With (5.6), equation (5.4) is reduced to

$$
\frac{H k}{D_{C J}} \frac{Q}{D_{C J}^{2}}=\exp \left(1.78 \theta_{C J}\right) \frac{9 \tan \alpha}{3-\gamma} \frac{(\gamma-1)^{2}}{(\gamma+1)^{3}} .
$$

As a consistency check, we verify that substitution of the values of $\alpha, Q, k, H, D_{C J}$ and $\gamma$ leads to $\theta_{C J_{c}}=4.0$. This value is close to the actual critical reduced activation energy. From figure 4, we see that $\theta_{C J_{c}}$ is between 3.5 (success) and 3.75 (failure) when $H=36.67$. 


\section{Conclusions}

In this work, we have identified modes of detonation diffraction that depend on the activation energy of a single-step irreversible Arrhenius reaction model. For the fixed value of gap half-width, $H=36.67$, we found three regimes of diffraction that resemble the super-, sub- and near-critical diffraction observed in experiments. Then, we extended the technique used by Eckett et al. (2000) to record the time derivative of temperature along the paths of particles that are close to ignition failure. We found that large ignition delays, and ultimately, local decoupling of the shock front from the reaction zone occur if the Lagrangian derivative of temperature vanishes immediately after the passage of the shock. We identified this condition, when flow field unsteadiness (as seen by the fluid particle) balances the rate of the energy release, as the critical condition for a failing detonation diffraction.

The importance of flow unsteadiness in the reaction zone structure equation is more apparent when comparing measured values of detonation speed and curvature with the $D_{n}-\kappa$ diagram derived from asymptotic quasi-steady quasi-one-dimensional theory (Stewart \& Bdzil 1988). We found that, in the case $\theta_{C J}=1$, the measured front curvature and detonation speed approach the asymptotic $D_{n}-\kappa$ values only when the wavefront has moved past a distance of the order of $6 \mathrm{H}$ from the corner. In the case $\theta_{C J}=3.5$, we did not observe a quasi-steady state before simulation termination. An even clearer indication of flow unsteadiness is provided by the maximum measured front curvature at the channel centre in figure 6. In the near-critical case $\theta_{C J}=3.5$, immediately after the arrival of the corner disturbance, front curvature rapidly reaches a value ten times larger than the maximum curvature allowed by a cylindrical steadystate detonation with the same activation energy. Even with this large value of detonation curvature, we found that the large-scale features of the wavefront can be considered quasi-one-dimensional. This can be seen in figure 6 , where the minimum measured wavefront radius of curvature (at the channel centre), $1 / \kappa_{a}>H / 4.1=9.1$, is sufficiently larger than unity.

In summary, our simulations indicate that a diffracting detonation wavefront is not insulated from transverse perturbations due to unsteady rarefaction waves. This appears in the propagation angle of the head disturbance with respect to the undisturbed front and, when local re-ignition takes place, in the complex interaction of the reflected expansion front with the partially decoupling detonation structure. In fact, sonic conditions are not reached at the end of the reaction zone for a relatively long transient, even in cases where detonation transmission is successful. This limits the applicability of the quasi-steady model by Stewart \& Bdzil (1988) when studying the critical diameter problem for mixtures whose reaction rate strongly depends on temperature. In the case of sub-critical diffractions, our simulations do not indicate any direct role played by front curvature. Rather, unsteadiness, due to deceleration of the lead shock, is found to be the dominant negative forcing factor that drives the ignition delay. Our conclusions disagree with the global failure mechanism proposed by Lee (1996) for mixtures with relatively small activation energies, as this mechanism postulates the existence of a critical curvature to explain detonation failure.

Our work addresses the purely gasdynamic quenching mechanisms, since the detonation cellular structure was not treated in the present simulations. The parameter range and initial conditions were chosen deliberately so that cellular structure was initially suppressed. The decision to carry out the computations without cellular structure was taken to facilitate shock tracking and the order-of-magnitude analysis along particle paths. Clearly, the result obtained from this analysis depends, in part, on this simplification. 
Our model is also limited to the description of a system governed by very simple reaction kinetics. It is an open issue whether the current results provide a lower bound for critical diffraction conditions in the presence of cellular structure and with a more detailed reaction mechanism. This is a significant extension of the present study, and we have left it for future investigation. Since the present parallel computations involved week-long simulations and stretched to a limit the capability of analysing large volumes of data, we anticipate that these investigations will require implementation of parallel adaptive mesh refinement algorithms and chemical kinetics reduction techniques.

Even if the empirical relationships between cell detonation width and critical tube diameter (or gap width, in two dimensions) are not applicable in the present case, studies by Westbrook \& Urtiew (1983), Moen et al. (1982, 1984), and Schultz (2000) have demonstrated that equally reasonable correlations exist between critical tube diameter, reaction zone length and activation energy. At present, there are no reliable methods for computing cell width, except for mixtures with very weak instabilities, but some progress can be made by considering the role of the reaction zone structure directly. Starting from similar considerations of the role played by unsteadiness, Schultz (2000) was successful in connecting the available experimental data with a critical model of spherical detonation diffraction leading to an equation similar to (5.7).

This work was carried out at the Caltech ASC 'Centre for Simulation of Dynamic Response of Materials' and funded by Contract B341492 under DOE Contract W-7405-ENG-48.

\section{Appendix A. Shock-tracking algorithm}

Detonation speed, shock acceleration and front curvature of the detonation wave are reconstructed in a post-processing step from shock-tracking data collected during the simulation. Shock tracking is performed in a non-intrusive step at each time integration after updating the flow field. The tracking algorithm consists of a sequence of sweeps of the current solution in the $x$ and $y$ coordinate directions. For each sweep, the position of the first peak of density that emerges from the undisturbed flow is searched. The shock location is taken as the position of the flex point in the numerical representation of the shock. The flex is defined as the midpoint between the peak value of density and the undisturbed value. Its position is estimated as a linear interpolation between the two grid points that bracket this value.

In the post-processing step at the end of the simulation, quantities such as the normal detonation velocity, $D_{n}$, and the local curvature, $\kappa$, are evaluated through finite-difference approximations for spatial and temporal derivatives of the shock position. For instance, the curvature along the channel plane of symmetry requires the evaluation of a second-order derivative, which we compute as a centred difference scheme,

$$
\kappa_{a}=2 \frac{\bar{x}_{m-w}-\bar{x}_{m}}{w^{2} \Delta y^{2}}+C w^{2} \Delta y^{2},
$$

where $C$ depends on the truncation error. In the equation above, we skipped $w$ grid points in both directions from $y_{m}$, and then used the symmetry condition for the tracked shock positions $\bar{x}_{m+w}=\bar{x}_{m-w}$. A similar formula is used for evaluating the curvature along the corner wall. 
Particular care is required to control the error associated with the approximation of derivatives of the shock position. Errors due to linear interpolation at the flex point in the shock profile and to high-frequency oscillations of the post-shock state (occurring when the wavefront crosses the interface between computational cells) appear in the numerator of (A 1). Since these errors can be of the same order as the grid spacing, a naive implementation of this formula may not converge under grid refinement. Aslam, Bdzil \& Hill (1998) raised the issue and suggested increasing the number of skipped points $w$ with grid refinement in such a way that the difference in (A 1) is never too small compared with the position error. In a convergence study in Arienti (2002), different strategies for choosing the points for differentiation are compared. Following that study, in the present work, we present results where $w=16$ in (A 1) for the coarse-resolution study and $w=21$ for the high-resolution study.

\section{Appendix B. Lagrangian particles integration}

Integration of the particle trajectory is performed with a predictor-corrector method in the form of an Adams-Bashforth predictor (P) followed by an Adams-Moulton corrector (C). Only one PC iteration is performed. The overall scheme is of the PECE type, where step E indicates the update of the derivative part from the last computed value (in Press et al. 1992, pp. 747-751). Step P is computed before advancing the Euler equations by a time step, and step C is computed after. A master-slave strategy (see, for instance, Wilkinson \& Allen 1999) is implemented to track particles that are transported from one processor sub-domain to the next.

Particle data analysis starts only after the first peak value in particle pressure data. Lagrangian derivatives are directly computed by finite-difference operations on the particle data stream, whereas partial time derivatives (in the intrinsic reference frame) are available only indirectly from the relation

$$
\partial /\left.\partial t\right|_{\xi, \eta}=D / D t-w_{\eta} \partial / \partial \eta-w_{\xi} \partial / \partial \xi
$$

The spatial gradients in $\eta$ and $\xi$ are obtained by coordinate transformation from the stored gradients in $x$ and $y$. When the partial time derivative is small compared to $\mathrm{D} / \mathrm{D} t$ (a limit case is a ZND detonation where $\partial /\left.\partial t\right|_{\xi, \eta}$ is identically zero), equation (B 1) is prone to cancellation errors. Since the partial time derivatives are combined in the unsteady term as

$$
w_{\eta}\left(D_{n}-w_{\eta}\right)_{, t}+\frac{P_{, t}}{\rho},
$$

an alternative solution is to evaluate this entire term by rearranging momentum and mass conservation equations. Under conditions of symmetry, the result

$$
w_{\eta}\left(D_{s}-w_{\eta}\right)_{, t}+\frac{P_{, t}}{\rho}=\frac{1}{\rho}\left(\frac{\mathrm{D} P}{\mathrm{D} t}-w_{\eta}^{2} \frac{\mathrm{D} \rho}{\mathrm{D} t}\right)+w_{\eta}^{2} \frac{\kappa_{s}\left(D_{s}-w_{\eta}\right)}{1-\eta \kappa_{s}}+w_{\eta}^{2} \frac{w_{\xi, \xi}}{1-\eta \kappa_{s}}
$$

is numerically easier to treat and is, therefore, used in (2.13) to evaluate the unsteady terms.

\section{REFERENCES}

ArIENTI, M. 2002 A numerical and analytical study of detonation diffraction. PhD thesis, California Institute of Technology.

Arienti, M., Hung, P., Morano, E. O. \& Shepherd, J. E. 2003 A level set approach to EulerianLagrangian coupling. J. Comput. Phys. 185, 213-251. 
Aslam, T. D., Bdzil, J. B. \& Hill, L. 1998 Extension to DSD theory: analysis of PBX 9502 rate stick data. In 11th Symp. (Intl) on Detonation, pp. 21-29. Office of Naval Research.

Barthel, H. O. \& Strehlow, R. A. 1966 Wave propagation in one-dimensional reactive flows. Phys. Fluids A 9, 1896-1907.

BdziL, J. B. \& Aslam, T. D. 2000 Detonation front models: theories and methods. LA-UR-00-942. Los Alamos National Laboratory.

BDzIL, J. B. \& STEwART, D. S. 1989 Modeling two-dimensional detonations with detonation shock dynamics. Phys. Fluids A 1, 1261-1267.

Desbordes, D. 1988 Transmission of overdriven plane detonations: critical diameter as a function of cell regularity and size. Prog. Astronaut. Aeronaut. 114, 170-185.

ECKETT, C. A. 2001 Numerical and analytical studies of the dynamics of gaseous detonations. PhD thesis, California Institute of Technology.

Eckett, C. A., Quirk, J. J. \& Shepherd, J. E. 2000 The role of unsteadiness in direct initiation of gaseous detonations. J. Fluid Mech. 421, 147-183.

Edwards, D. H., Thomas, G. O. \& Nettleton, M. A. 1979 The diffraction of a planar detonation wave at an abrupt area change. J. Fluid Mech. 95, 79-96.

Fickett, W. \& Davis, W. C. 1979 Detonation. University of California Press.

Guirao, C. M., Knystautas, R. \& Lee, J. H. 1987 A summary of hydrogen-air detonation experiments. Tech. Rep. SAND87-7128. Sandia National Laboratories.

Harten, A. 1983 High resolution schemes for hyperbolic conservation laws. J. Comput. Phys. 49, 357-393.

Helzel, C., Leveque, R. J. \& Warnecke, G. 2000 A modified fractional step method for the accurate approximation of detonation waves. SIAM J. Sci. Comput. 22, 1489-1510.

Jones, D. A., Kemister, G., Oran, E. S. \& Sichel, M. 1996 The influence of cellular structure on detonation transmission. Shock Waves 6, 119-129.

Jones, D. A., Kemister, G., Tonello, N. A., Oran, E. S. \& Sichel, M. 2000 Numerical simulation of detonation reignition in $\mathrm{H}_{2}-\mathrm{O}_{2}$ mixtures in area expansions. Shock Waves 10, 33-41.

Jones, D. A., Sichel, M., Guirguis, R. \& Oran, E. S. 1991 Numerical simulation of layered detonations. Prog. Astronaut. Aeronaut. 133, 202-219.

Jones, D. A., Sichel, M. \& Oran, E. S. 1995 Reignition of detonation by reflected shocks. Shock Waves 5, 47-57.

Jones, D. A., Sichel, M., Oran, E. S. \& Guirguis, R. 1990 Detonation transmission in layered explosives. In 23rd Symp. (Intl) on Combustion, pp. 1805-1811. The Combustion Institute.

Knystautas, R., Lee, J. H. \& Guirao, C. M. 1982 The critical tube diameter for detonation failure in hydrocarbon-air mixtures. Combust. Flame 48, 63-83.

Lee, H. I. \& Stewart, D. S. 1990 Calculation of linear detonation instability: one-dimensional instability of plane detonation. J. Fluid Mech. 216, 103-132.

LEE, J. H. S. 1996 On the critical diameter problem. In Dynamics of Exothermicity (ed. J. R. Bowen), pp. 321-336. Gordon \& Breech.

Li, C. \& Kailasanath, K. 2000 Detonation transmission and transition in channels of different sizes. In 28th Symp. (Intl) on Combustion, pp. 603-609. The Combustion Institute.

Liu, J. C., Liou, J., Sichel, M. \& Nichols, C. W. K. J. A. 1987 Diffraction and transmission of a detonation into a bounding explosive layer. In 21st Symp. (Intl) on Combustion, pp. 1639-1647. The Combustion Institute.

Liu, J. C., Sichel, M. \& Kauffmann, C. W. 1988 The lateral interaction of detonating and detonable gas mixtures. Prog. Astronaut. Aeronaut. 114, 264-283.

Menikoff, R., Lackner, K. S. \& Bukiet, B. G. 1996 Modeling flows with curved detonation waves. Combust. Flame 104, 219-240.

Mitrofanov, V. V. \& Soloukhin, R. I. 1965 The diffraction of multifront detonation waves. Sov. Phys. Dokl. 9, 1055-1058.

Moen, I., Funk, J. W., Ward, S. A., Rude, G. M. \& Thibault, P. A. 1984 Detonation length scales for fuel-air explosives. Prog. Astronaut. Aeronaut. 94, 55-79.

Moen, I., Murray, S., Buerketvedt, D., Rinnan, A., Knystautas, R. \& Lee, J. 1982 Diffraction of detonation from tubes into a large fuel-air explosive cloud. In 19th Symp. (Intl) on Detonation, pp. 635-644.

Oran, E. S., Boris, J., Jones, D. A. \& Sichel, M. 1993 Ignition in a complex Mach structure. Prog. Astronaut. Aeronaut. 153, 241-252. 
Oran, E. S., Jones, D. A. \& Sichel, M. 1992 Numerical simulations of detonation transmission. Proc. R. Soc. Lond. A 436, 267-297.

Parashar, M., Browne, J. C., Edwards, C. \& Klimkowski, K. 1997 A common data management infrastructure for adaptive algorithms for PDE solutions. Proc. 1997 ACM/IEEE Conf. on Supercomputing, pp. 1-22. ACM Press (CDROM).

PARAShaR, M. \& BRowne, J. C. 2000 System engineering for high performance computing software: The HDDA/DAGH Infrastructure for implementation of parallel structured adaptive mesh refinement. IMA: Structured Adaptive Mesh Refinement (SAMR) Grid Methods, vol. 117, pp. 1-18. Springer.

Press, W. H., Teukolsky, S. A., Vetterling, W. T. \& Flannery, B. P. 1992 Numerical Recipes: the Art of Scientific Computing, pp. 747-752. Cambridge University Press.

Radulescu, M. I., Higgins, A. J., Murray, S. B. \& Lee, J. H. S. 2003 An experimental investigation of the direct intitiaion of cylindrical detonations. J. Fluid Mech. 480, 1-24.

REYNOLDS, W. C. 1986 The element potential method for chemical equilibrium analysis: implementation in the interactive program STANJAN. Tech. Rep. Stanford University, Dept of Mech. Engng.

RoE, P. L. 1986 Characteristic-based schemes for the Euler equations. Annu. Rev. Fluid Mech. 18, $337-365$.

Schultz, E. 2000 Detonation diffraction through an abrupt area expansion. PhD thesis, California Institute of Technology.

Sharpe, G. J. 2001 Transverse waves in numerical simulations of cellular detonations. J. Fluid Mech. 447, 31-51.

Shepherd, J. E., Moen, I. O., Murray, S. B. \& Thibault, P. A. 1986 Analyses of the cellular structure of detonation. In 21st Symp. (Intl) on Combustion, pp. 1649-1658. The Combustion Institute.

Skews, B. W. 1967 The shape of a diffracting shock wave. J. Fluid Mech. 29, 297-304.

Snir, M., Otto, S. W., Huss-Lederman, S., Walker, D. W. \& Dongarra, J. 1996 MPI: The Complete Reference. Scientific and Engineering Computation. MIT Press.

Stewart, D. S. \& BDziL, J. B. 1988 The shock dynamics of stable multi-dimensional detonation. Combust. Flame 72, 311-323.

Stewart, D. S. \& YaO, J. 1998 The normal detonation shock velocity-curvature relationship for materials with nonideal equation of state and multiple turning points. Combust. Flame 113, 224-235.

Strang, G. 1968 On the construction and comparison of difference schemes. SIAM J. Numer. Anal. 5, 506-517.

Strehlow, R. A. \& Fernandes, F. D. 1965 Transverse waves in detonations. Combust. Flame 9, $109-119$.

Westbrook, C. K. \& Urtiew, P. A. 1983 Use of chemical kinetics to predict critical parameters of gaseous detonations. Combust. Explosion Shock Waves 19, 753-766.

Whitham, G. B. 1974 Linear and Nonlinear Waves. Wiley.

Wilkinson, B. \& Allen, M. 1999 Parallel Programming, Techniques and Applications Using Networked Workstations and Parallel Computers. Prentice Hall.

Williams, D. N., Bauwens, L. \& Oran, E. S. 1996 Detailed structure and propagation of threedimensional detonations. In 26th Symp. (Intl) on Combustion, pp. 2991-2998. The Combustion Institute.

Xu, S., Aslam, T. \& Stewart, D. S. 1997 High resolution numerical simulation of ideal and nonideal compressible reacting flows with embedded internal boundaries. Combust. Theor. Model. 1, 113-142.

YaO, J. \& Stewart, D. S. 1995 On the normal shock velocity-curvature relationship for materials with large activation energy. Combust. Flame 100, 519-528.

Zel'dovich, I. B., Kogarko, S. M. \& Simonov, N. N. 1956 An experimental investigation of spherical detonation of gases. Sov. Phys. Tech. Phys. 1, 1689-1713. 